Search for sub-eV axion-like resonance states via stimulated quasi-parallel laser collisions with the parameterization including fully asymmetric collisional geometry

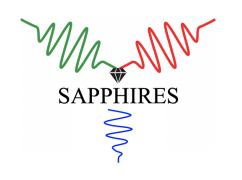

\title{
The SAPPHIRES collaboration
}

Kensuke Homma, ${ }^{a, 1,2}$ Yuri Kirita, ${ }^{a, 1}$ Masaki Hashida, ${ }^{b, c, 3}$ Yusuke Hirahara, ${ }^{a}$ Shunsuke Inoue, ${ }^{b, c}$ Fumiya Ishibashi, ${ }^{a}$ Yoshihide Nakamiya, ${ }^{b, d}$ Liviu Neagu, ${ }^{d, e}$ Akihide Nobuhiro, ${ }^{a}$ Takaya Ozaki, ${ }^{a}$ Madalin-Mihai Rosu, ${ }^{d}$ Shuji Sakabe ${ }^{b, c}$ and Ovidiu Tesileanu ${ }^{d}$

${ }^{a}$ Graduate School of Advanced Science and Engineering, Hiroshima University, Kagamiyama, Higashi-Hiroshima 739-8526, Japan

${ }^{b}$ Institute for Chemical Research, Kyoto University, Uji, Kyoto 611-0011, Japan

${ }^{c}$ Graduate School of Science, Kyoto University, Sakyouku, Kyoto 606-8502, Japan

${ }^{d}$ Extreme Light Infrastructure-Nuclear Physics (ELI-NP) and Horia Hulubei National Institute for RED in Physics and Nuclear Engineering (IFIN-HH), 30 Reactorului St., P.O. Box MG-6, Bucharest-Magurele, Judetul Ilfov RO-077125, Romania e National Institute for Laser, Plasma and Radiation Physics, 409 Atomistilor, P.O. Box MG-36, Magurele 07r125, Judetul Ilfov, Romania

E-mail: khomma@hiroshima-u.ac.jp, kirita@quark.hiroshima-u.ac.jp, hashida.masaki.3z@kyoto-u.ac.jp, hirahara@quark.hiroshima-u.ac.jp, sinoue@laser.kuicr.kyoto-u.ac.jp, isibasi@quark.hiroshima-u.ac.jp, yoshihide.nakamiya@eli-np.ro, liviu.neagu@eli-np.ro, nobuhiro@quark.hiroshima-u.ac.jp, ozaki@quark.hiroshima-u.ac.jp, madalin.rosu@eli-np.ro, sakabe@laser.kuicr.kyoto-u.ac.jp, ovidiu.tesileanu@eli-np.ro

\footnotetext{
${ }^{1}$ Co-first author.

${ }^{2}$ Corresponding author.

${ }^{3}$ Now at Research institute of Science and Technology, Tokai University, 4-1-1 Kitakaname, Hiratsuka, Kanagawa 259-1292, Japan.
} 
ABSTRACT: We have searched for axion-like resonance states by colliding optical photons in a focused laser field (creation beam) by adding another laser field (inducing beam) for stimulation of the resonance decays, where frequency-converted signal photons can be created as a result of stimulated photon-photon scattering via exchanges of axion-like resonances. A quasi-parallel collision system (QPS) in such a focused field allows access to the sub-eV mass range of resonance particles. In past searches in QPS, for simplicity, we interpreted the scattering rate based on an analytically calculable symmetric collision geometry in both incident angles and incident energies by partially implementing the asymmetric nature to meet the actual experimental conditions. In this paper, we present new search results based on a complete parameterization including fully asymmetric collisional geometries. In particular, we combined a linearly polarized creation laser and a circularly polarized inducing laser to match the new parameterization. A $0.10 \mathrm{~mJ} / 31 \mathrm{fs}$ Ti:sapphire laser pulse and a $0.20 \mathrm{~mJ} / 9 \mathrm{~ns} \mathrm{Nd}$ :YAG laser pulse were spatiotemporally synchronized by sharing a common optical axis and focused into the vacuum system. Under a condition in which atomic background processes were completely negligible, no significant scattering signal was observed at the vacuum pressure of $2.6 \times 10^{-5} \mathrm{~Pa}$, thereby providing upper bounds on the coupling-mass relation by assuming exchanges of scalar and pseudoscalar fields at a $95 \%$ confidence level in the sub-eV mass range.

KEYwORDs: Dark matter, Particle and resonance production, Photon production, Other experiments, Exotics

ARXIV EPrint: 2105.01224 


\section{Contents}

1 Introduction 1

2 Formulae for obtaining $m-g / M$ relation numerically in general QPS geometry

2.1 Induced signal yield $\mathcal{Y}_{c+i}$

2.2 Vertex factors in scattering amplitude $\mathcal{M}_{\mathrm{S}} \quad 7$

2.3 Outline of numerical calculations 9

3 Experimental setup $\quad 10$

4 Data analysis 12

4.1 Counting number of photons by means of a peak finder 12

$\begin{array}{lll}4.2 & \text { Pressure dependence of atomic four-wave mixing process } & 13\end{array}$

$\begin{array}{lll}4.3 & \text { Focal-point stability } & 15\end{array}$

$\begin{array}{lll}4.4 & \text { Effective energy fraction in Gaussian beams } & 16\end{array}$

5 Search result $\quad 16$

6 Upper limits on coupling-mass relation for ALP exchanges 17

$\begin{array}{lll}7 & \text { Conclusions } & 20\end{array}$

A Pulse overlapping factor with different beam diameters 22

\section{Introduction}

Spontaneous symmetry breaking is the key concept for understanding the fundamental laws of physics. In particular, when a symmetry is global, the appearance of a massless Nambu-Goldstone boson (NGB) $[1,2]$ may be expected as a result of the broken symmetry. This viewpoint can be a robust guiding principle for predicting new particle states based on various types of global symmetries in different theoretical contexts, including axion [3$5,9,10]$, dilaton [11], inflaton [12], and string-inspired models [13-15]. However, NGBs are observed as pseudo-NGB states (pNGB) with finite masses due to complicated quantum corrections, such as pions in the context of quantum chromodynamics (QCD). If pNGBs are coupled only very weakly to matter, they could be natural candidates to explain the dark components of the universe [6-8]. When pNGB masses are relatively small and the couplings to matter are feeble, they are referred to herein as axion-like particles (ALPs). Although ALP masses are supposed to be small, how small is not known theoretically. Therefore, experimental efforts to search for ALPs over wide low-mass and weak-coupling domains are valuable for discovering some of the dark components of the universe. 
The XENON1T experiment recently reported an excess of electron recoil events compared with the defined background level [16]. Among three possible scenarios to explain this excess, the solar axion interpretation is the one that fits most nicely with the recoiled electron energy spectrum with $3.5 \sigma$ significance. The consistent axion mass range is $\mathcal{O}(0.1-10) \mathrm{eV}$ in the photon-axion coupling range of $\mathcal{O}\left(10^{-12}-10^{-9}\right) \mathrm{GeV}^{-1}$ depending on the electron-axion coupling and the QCD axion models [17-20]. However, there is strong tension between this interpretation and the existing constraints from stellar cooling [21-25]. If the aforementioned significance level further increases through improved observations in the near future and the solar axion scenario remains the one that is most valid, then this tension becomes a real issue. To resolve this issue, model-independent observations of the direct production of axions and their decay in laboratory experiments would be indispensable.

In this paper, we focus on the coupling between sub-eV ALPs and laser photons. To describe the coupling of scalar $(\phi)$ or pseudoscalar $(\sigma)$ ALPs to two photons, the following two effective Lagrangians are considered:

$$
-L_{\phi}=g M^{-1} \frac{1}{4} F_{\mu \nu} F^{\mu \nu} \phi, \quad-L_{\sigma}=g M^{-1} \frac{1}{4} F_{\mu \nu} \tilde{F}^{\mu \nu} \sigma,
$$

where $g$ is a dimensionless constant for a given energy scale $M$ at which a relevant global symmetry is broken, and $F^{\mu \nu}=\partial^{\mu} A^{\nu}-\partial^{\nu} A^{\mu}$ is the electromagnetic field strength tensor and its dual $\tilde{F}^{\mu \nu} \equiv \varepsilon^{\mu \nu \alpha \beta} F_{\alpha \beta}$ with the Levi-Civita symbol $\varepsilon^{i j k l}$.

Focusing on sub-eV ALPs, we have proposed to utilize quasi-parallel collision system (QPS) between two photon pairs with equal energy $\omega$ by combining and focusing twocolor lasers along a common optical axis [26] as illustrated in figure 1. The corresponding center-of-mass system (CMS) energy in the QPS is expressed as

$$
E_{\mathrm{CMS}}=2 \omega \sin \vartheta
$$

where $2 \vartheta$ is the relative angle between a pair of incident photons. By controlling the beam diameter $(d)$ and focal length $(f)$ experimentally, the QPS can be sensitive to ALP resonance states with the mass range of $0<m<2 \omega \sin \Delta \vartheta$, where $m$ is the ALP mass and $\Delta \vartheta$ can be adjusted by the focusing geometry determined with $\Delta \vartheta \sim(d / 2) / f$.

The first key feature of the proposed method [26] is the resonant ALP production via the $s$-channel exchange within the $E_{\mathrm{CMS}}$ uncertainty, which drastically enhances the production rate [26]. The second key feature is stimulated decays of produced ALPs to fixed final states via energy-momentum conservation between four photons in the initial and final states. This stimulated resonant scattering rate eventually becomes proportional to the square of the number of photons in the creation laser beam and to the number of photons in the inducing laser beam. This cubic dependence on the number of photons in the beams offers opportunities to search for ALPs with extremely weak coupling when the beam intensity is high enough [27].

In past searches [28-30], we provided constraints on the coupling-mass relation based on a symmetric QPS interpretation in which the incident angles and energies of the two initial-state photons are symmetric (figure 1). Based on this parameterization, we provided 


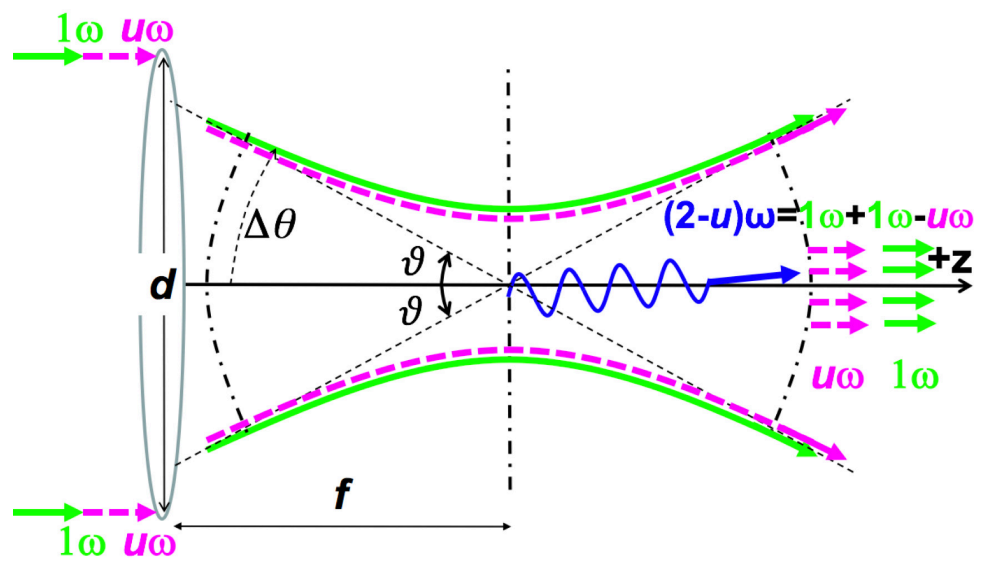

Figure 1. Concept of stimulated resonant photon-photon scattering in a quasi-parallel collision system (QPS) by focusing two-color laser fields in a vacuum. This figure is quoted from [28] with a slight modification. A coherent field with energy $\omega$ (solid green line) is combined with a different-color coherent field with energy $u \omega(0<u<1)$ (dashed red line). The combined fields are focused by a lens element in a vacuum. Emission of signal photons with energy $(2-u) \omega$ (blue wavy line) is stimulated as a result of energy-momentum conservation in the scattering process $\omega+\omega \rightarrow \phi / \sigma \rightarrow(2-u) \omega+u \omega$ via a resonance state $\phi / \sigma$. Given the focusing parameters of beam diameter $d$ and focal length $f$, the incident angle $\vartheta$ is expected to vary over $0<\vartheta \leq \Delta \theta$. This unavoidable ambiguity of the wave vectors of the incident light waves provides a wide window for accessing different center-of-mass system energies at a given point in time.

conservative constraints, and we respected the simple analytic treatment with symmetric collisions because the initial search was made with narrow-bandwidth lasers. However, in a short-pulse laser that is close to the Fourier-transform limit, where the relation between laser frequency and time duration is governed by the wavelike nature of the system (i.e., the uncertainty principle), we must accept an energy spread in principle, and so the approximation of symmetric incident energies is not realistic. In addition, at the diffraction limit where the beam diameters reach their minimum values, the incident angles must also fluctuate greatly because of momentum-position uncertainties. Therefore, we must accept a situation in which the incident photon energies and angles are both asymmetric. Recently, we formulated the interaction rate based on the fully asymmetric collision system [27], where the non-coaxial geometry of the two-photon collisions and stimulated decays are explicitly included with respect to a given coaxial geometry of focused beams (figure $2 b$ ).

In general, linear polarization states in laser beams are supposed to be fixed precisely. However, linear polarization states around a focal point are not simple plane-wave states because the directions of the wave vectors are not identical in three dimensions, as illustrated in figure 3 , even if the wave vectors are all aligned to a unique direction before focusing. If we require the transverse wave states of individual light waves, then the polarization directions of individual light waves must fluctuate depending on the individual wave vector directions. Therefore, these fluctuations must be included in both the creation and inducing lasers around the focal points. In order for the stimulation due to the associated inducing field to be effective, one of the final-state light waves must coincide with the mo- 


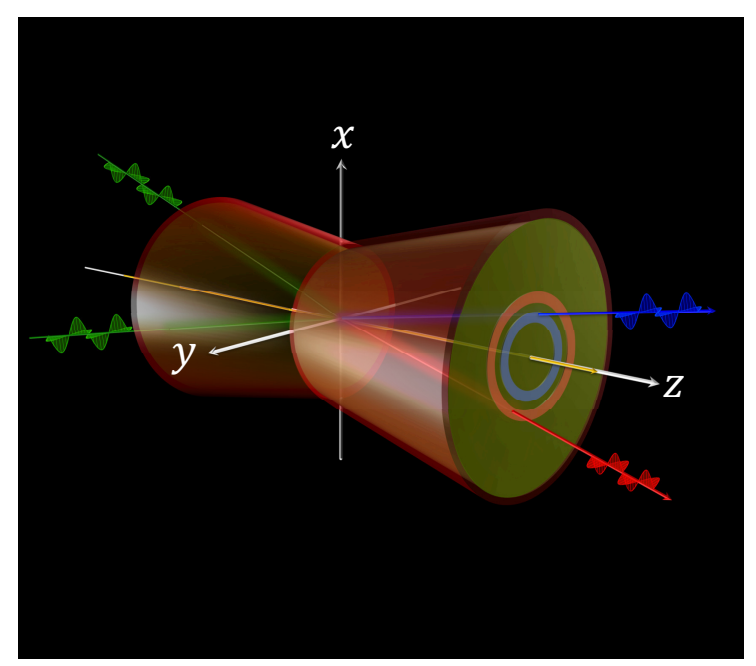

(a)

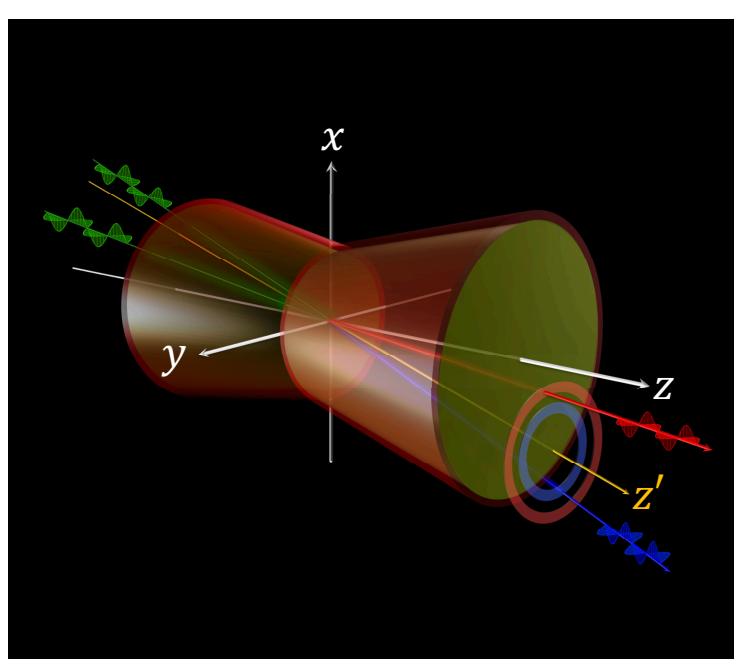

(b)

Figure 2. Classification of collision geometries in QPS. The two figures are extracted from figure 1 of [27]. (a) Symmetric incidence and coaxial scattering, where the incident angles of the two wave vectors and their energies are symmetric, and the transverse momentum of the photon pair, $p_{T}$, always vanishes with respect to the common optical axis $z$. (b) Asymmetric incidence and non-coaxial scattering, where the incident angles of the two wave vectors and their energies are asymmetric, resulting in a finite value of $p_{T}$ with respect to the common optical axis $z$. The zero$p_{T}$ axis $\left(z^{\prime}\right.$-axis) is always configurable for arbitrary pairs of incident wave vectors.

mentum and also with the linear polarization state of the inducing laser waves. Therefore, fluctuations of linear polarization directions make the evaluation of the stimulation effect in non-coaxial scattering events very complicated because coaxial symmetry of the inducing laser beam is no longer applicable. However, if a collection of light waves is circularly polarized, we can avoid this complication because even if the directions of the waves in three dimensions are changed, the individual light waves retain the circular polarization. Therefore, in [27] we evaluated the stimulation effect based on circularly polarized laser beams. However, there is an experimental constraint in that high-intensity laser pulses are provided as linearly polarized states, and changing their polarization from linear to circular adds a technical difficulty. In this paper, we report new results for sub-eV ALP searches by combining linearly and circularly polarized states for the creation and inducing lasers, respectively, based on the parameterization including the fully asymmetric collision geometry in QPS. This is in contrast to previous works in which we reported searches based on the symmetric QPS approximation with linear polarization states for both the creation and inducing lasers [28-30].

\section{Formulae for obtaining $m-g / M$ relation numerically in general QPS geometry}

Stimulated resonant photon-photon scattering in the most general collisional geometry including asymmetric incidence and non-coaxial scattering was formulated in [27], and 


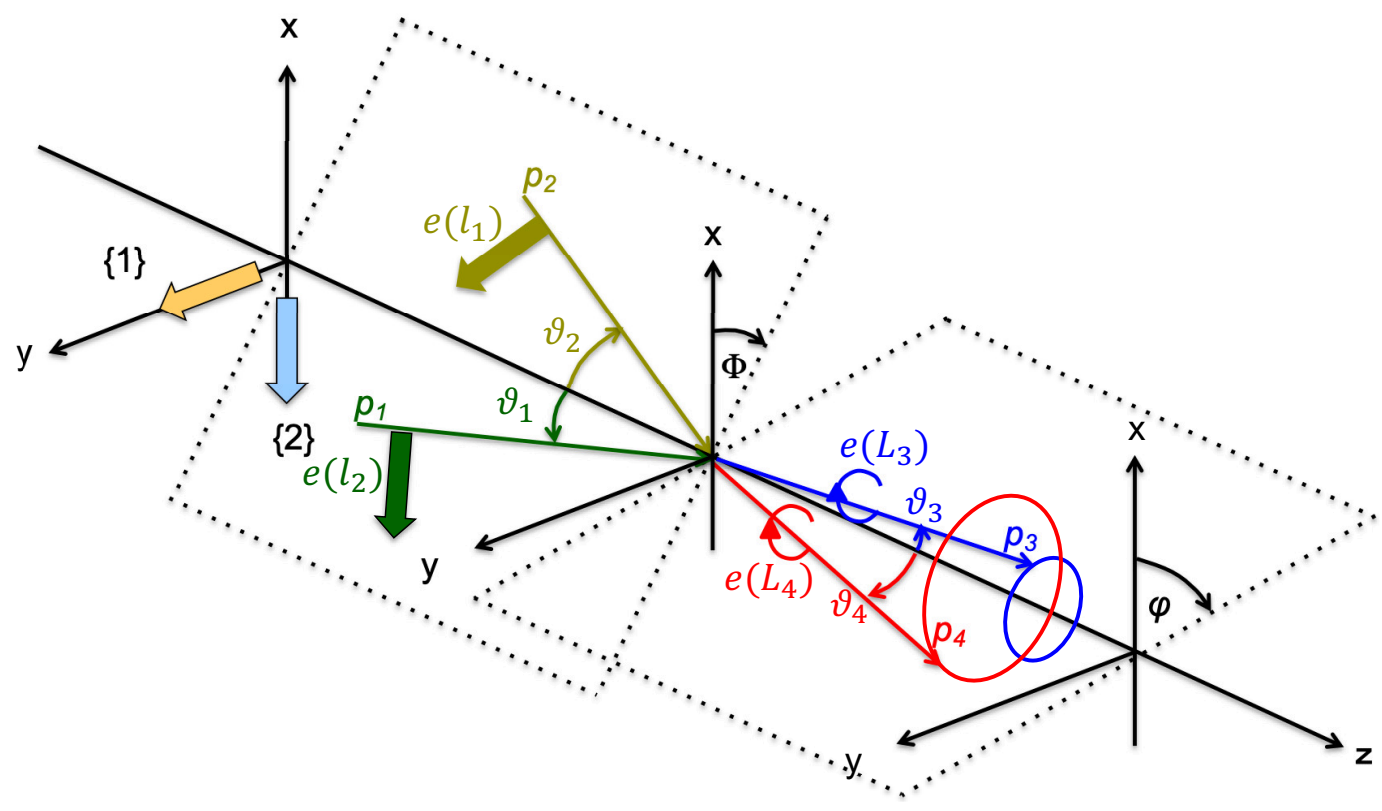

Figure 3. Definitions of four-momentum vectors $p_{i}$ and four-polarization vectors $e\left(\lambda_{p_{i}}\right)$ with polarization states $\lambda_{p_{i}}$ for the initial-state $(i=1,2)$ and final-state $(i=3,4)$ light waves. $l_{i}$ indicates mixture of experimentally defined orthogonal linear polarization states $\{1\}$ and $\{2\}$ before focusing in laboratory coordinates, and $L_{i}$ indicates left-handed circular polarization states. These polarization vectors are mapped on the rotating reaction planes in the case of asymmetric incident angles and energies in QPS, where the transverse momentum $p_{T}$ of the $p_{1}+p_{2}$ pair is constraint to be zero as a specially simple case of general asymmetric collisions.

the full details can be found in the appendix of [27]. In the following subsections, we briefly explain how to relate the physical parameters of mass $m$ and coupling $g / M$ with the observed number of stimulated photon signals by reviewing only the relevant formulae to discuss the interaction rate dedicated for this search. We specifically replace the vertex factors in the scattering amplitude because we must change from the circular polarization formulated in [27] to the linear one for both scalar and pseudoscalar field exchanges in this search.

We address a search for signal photons $p_{3}$ for the following degenerate case in the generic QPS:

$$
\left\langle p_{c}\left(p_{1}\right)\right\rangle+\left\langle p_{c}\left(p_{2}\right)\right\rangle \rightarrow p_{3}+\left\langle p_{i}\left(p_{4}\right)\right\rangle,
$$

where \langle\rangle indicates that $p_{1}$ and $p_{2}$ are chosen stochastically from a single focused coherent beam whose central four-momentum is $p_{c}$ for the creation of ALPs via $s$-channel photonphoton scattering, while the focused coherent beam with the central four-momentum $p_{i}$ is co-moving to induce emission of signal photons $p_{3}$ when a fraction of the $p_{i}$ beam coincides with $p_{4}$.

In symmetric incidence and coaxial scattering as illustrated in figure $2 \mathrm{a}$, transverse momenta of stochastically selected wave pairs, $p_{T}$, are constraint to be zero with respect to the common optical axis $z$. Given that the azimuthal angles of the final-state wave vectors are axially symmetric around the $z$-axis, the evaluation of the inducible momentum or 
angular range can be greatly simplified owing to the axial symmetric nature of the focused laser beams. In contrast, asymmetric incidence and non-coaxial scattering in figure $2 \mathrm{~b}$ introduces a finite transverse momentum. In this case, a new zero- $p_{T}$ axis, referred to as the $z^{\prime}$-axis, can be found for the pair of incident wave vectors. Based on the $z^{\prime}$-axis, the axial symmetric nature of the azimuthal angles of the final-state wave vectors can be restored. However, the inducing coherent field is still physically fixed to the common optical axis $z$. This situation complicates the evaluation of the inducible momentum range depending on an arbitrarily formed $z^{\prime}$-axis. To solve this complication, a numerical integration is required to express the number of signal photons in the scattering process (2.1) per pulse collision, $\mathcal{Y}_{c+i}$, as soon reviewed in the following subsections. With $\mathcal{Y}_{c+i}$ and a set of laser beam parameters $P$, the number of stimulated signal photons, $N_{\text {obs }}$, as a function of mass $m$ and coupling $g / M$ is eventually expressed as

$$
N_{\mathrm{obs}}=\mathcal{Y}_{c+i}(m, g / M ; P) t_{a} r \epsilon,
$$

where $t_{a}$ is the data acquisition time, $r$ is the repetition rate of the pulsed beams, and $\epsilon$ is the efficiency of detecting $p_{3}$. For a set of $m$ values and an $N_{\text {obs }}$, a set of coupling $g / M$ is evaluated by solving this equation numerically.

\subsection{Induced signal yield $\mathcal{Y}_{c+i}$}

With the average numbers of photons $N_{c}$ and $N_{i}$ for the creation and inducing coherent beams, respectively, and units given in [ ] with units of length $L$ and second $s$, the induced signal yield $\mathcal{Y}_{c+i}$ per pulse collision is evaluated as

$$
\begin{aligned}
\mathcal{Y}_{c+i}[1]= & \left(N_{c} / 2\right)\left(N_{c} / 2\right) N_{i} \times \\
& \left(\int_{-Z_{\mathrm{R}} / c}^{0} d t \int_{-\infty}^{+\infty} d \boldsymbol{r} \rho_{c}(\boldsymbol{r}, t) \rho_{c}(\boldsymbol{r}, t) \rho_{i}(\boldsymbol{r}, t) V_{i}\right) \times \\
& \left(\int d Q_{\mathrm{I}} W\left(Q_{\mathrm{I}}\right) \frac{c}{2 \omega_{1} 2 \omega_{2}}\left|\mathcal{M}_{s}\left(Q^{\prime}\right)\right|^{2} d L_{i p s}^{\prime I}\right) \\
\equiv & \frac{1}{4} N_{c}^{2} N_{i} \mathcal{D}_{\mathrm{I}}\left[s / L^{3}\right] \bar{\Sigma}_{\mathrm{I}}\left[L^{3} / s\right] .
\end{aligned}
$$

$\mathcal{D}_{\mathrm{I}}$ in eq. (2.3) is a spatiotemporal overlapping factor in laboratory coordinates (see $x, y, z$ in figure 3 ) of the focused creation beam (subscript $c$ ) with the co-moving focused inducing beam (subscript $i$ ) limited in the Rayleigh length $Z_{\mathrm{R}}$ only around the focal spot for a conservative evaluation. The following photon number densities $\rho_{k=c, i}$ based on the Gaussian beam parameterization are integrated over spacetime $(t, \boldsymbol{r})$ :

$$
\begin{aligned}
\rho_{k}(t, \boldsymbol{r})= & \left(\frac{2}{\pi}\right)^{\frac{3}{2}} \frac{1}{w_{k}^{2}(c t) c \tau_{k}} \times \\
& \exp \left(-2 \frac{x^{2}+y^{2}}{w_{k}^{2}(c t)}\right) \exp \left(-2\left(\frac{z-c t}{c \tau_{k}}\right)^{2}\right),
\end{aligned}
$$

where $w_{k}$ are the beam radii as a function of time $t$ whose origin is set at the moment when all the pulses reach the focal point, and $\tau_{k}$ are the time durations of the pulsed laser 
beams with the speed of light $c$ and the volume of the inducing beam $V_{i}$ defined as

$$
V_{i}=(\pi / 2)^{3 / 2} w_{i 0}^{2} c \tau_{i}
$$

where $w_{i 0}$ is the beam waist (minimum radius) of the inducing beam. The actually used overlapping factor configured for the case of different beam diameters between creation and inducing lasers is summarized in the appendix of this paper.

$\bar{\Sigma}_{\mathrm{I}}$ in eq. (2.3) is an integrated inducible volume-wise interaction rate that integrates the square of the scattering amplitude $\left|\mathcal{M}_{s}\left(Q^{\prime}\right)\right|^{2}$ over an inducible variable set comprising energies $\omega_{i}$, polar angles $\Theta_{i}$, and azimuthal angles $\Phi_{i}$ in laboratory coordinates for $i=$ $1,2,4: Q_{\mathrm{I}} \equiv\left\{Q, \omega_{4}, \Theta_{4}, \Phi_{4}\right\}$ with $Q \equiv\left\{\omega_{1}, \Theta_{1}, \Phi_{1}, \omega_{2}, \Theta_{2}, \Phi_{2}\right\}$ by weighting with multiple Gaussian distributions:

$$
W\left(Q_{\mathrm{I}}\right) \equiv \Pi_{i} G_{E}\left(\omega_{i}\right) G_{p}\left(\Theta_{i}, \Phi_{i}\right)
$$

with Gaussian distributions on energy $G_{E}$ and momentum $G_{p}$, and also over an inducible Lorentz-invariant phase space in zero- $p_{T}$ coordinates:

$$
d L_{i p s}^{\prime I}=(2 \pi)^{4} \delta\left(p_{3}^{\prime}+p_{4}^{\prime}-p_{1}^{\prime}-p_{2}^{\prime}\right) \frac{d^{3} p_{3}^{\prime}}{2 \omega_{3}(2 \pi)^{3}} \frac{d^{3} p_{4}^{\prime}}{2 \omega_{4}(2 \pi)^{3}}
$$

with two incident energies $\omega_{1}$ and $\omega_{2}$, where the primed variables are converted from $Q$ in laboratory coordinates to $Q^{\prime}$ in zero- $p_{T}$ coordinates via coordinate rotation. The inducing weight $W\left(Q_{\mathrm{I}}\right)$ takes care of the energy and momentum fractions of $p_{4}$ satisfying energy-momentum conservation with respect to the energy and momentum distributions of the given inducing laser beam in laboratory coordinates. The essential element of $\bar{\Sigma}_{\mathrm{I}}$ is the Lorentz-invariant scattering amplitude defined in zero- $p_{T}$ coordinates, $\mathcal{M}_{\mathrm{S}}\left(Q^{\prime}\right)$, for the given polarization states $S=a b c d$ in a two-body interaction: $p_{1}^{\prime}\{a\}+p_{2}^{\prime}\{b\} \rightarrow p_{3}^{\prime}\{c\}+p_{4}^{\prime}\{d\}$. Unless confusion is expected, for simplicity, we omit the prime symbol associated with the momentum vectors in the following explanations.

\subsection{Vertex factors in scattering amplitude $\mathcal{M}_{\mathrm{S}}$}

The polarization information is normally useful for distinguishing whether ALPs are scalar or pseudoscalar fields. When a two-body photon-photon scattering process $p_{1}+p_{2} \rightarrow p_{3}+p_{4}$ in four-momentum space occurs on an identical reaction plane, namely, when the coplanar condition $(\Phi=\varphi=0$ in figure 3$)$ is satisfied, the difference between scalar and pseudoscalar cases becomes distinct. Given an orthogonal set of linear polarization states $\{1\}$ and $\{2\}$, the non-zero scattering amplitudes are limited to the following cases:

$$
\begin{aligned}
& p_{1}\{1\}+p_{2}\{1\} \rightarrow p_{3}\{2\}+p_{4}\{2\}, \\
& p_{1}\{1\}+p_{2}\{1\} \rightarrow p_{3}\{1\}+p_{4}\{1\}
\end{aligned}
$$

for scalar field exchange and

$$
\begin{aligned}
& p_{1}\{1\}+p_{2}\{2\} \rightarrow p_{3}\{1\}+p_{4}\{2\}, \\
& p_{1}\{1\}+p_{2}\{2\} \rightarrow p_{3}\{2\}+p_{4}\{1\}
\end{aligned}
$$


for pseudoscalar field exchange, where swapping $\{1\}$ and $\{2\}$ gives the same scattering amplitudes.

However, as illustrated in figure 3 , the coplanar condition is, in most cases, not satisfied in QPS in contrast to CMS because the $\vec{p}_{1}-\vec{p}_{2}$ plane and the $\vec{p}_{3}-\vec{p}_{4}$ plane may differ from the $x-z$ plane defined with the laboratory coordinates. Therefore, we must introduce combinations of linear polarization states, $l_{1}$ and $l_{2}$, based on the theoretically introduced planes with respect to the experimental linear polarization states $\{1\}$ and $\{2\}$, which are mapped to the $y$ and $x$ axes, respectively. In the following search, we assign the P-polarized state of the creation laser to the $\{2\}$-state and combine it with the circularly polarized inducing laser. Note here that due to the rotating nature of the incident reaction plane in the focused geometry, even if the experimentally prepared linear polarization state is limited to $\{2\}$, polarization states defined on individual $\vec{p}_{1}-\vec{p}_{2}$ planes can contain both $\{1\}$ and $\{2\}$ components with different projection weights, resulting in sensitivities to both scalar and pseudoscalar fields. This situation is implemented quantitatively in the vertex factors as follows.

Based on expansion of the electromagnetic field strength tensor $F^{\mu \nu}$ and its dual $\tilde{F}^{\mu \nu}$, momentum-polarization tensors corresponding to the expanded coefficients are defined (see eqs. (A.5) and (A.6) in [27] for details). With a polarization four-vector $e_{i}\left(\lambda_{p}\right)$ with an arbitrary polarization state $\lambda_{p}$ associated with a four-momentum $p$, and the symbol * indicating the complex conjugate, the momentum-polarization tensors are defined as

$$
\begin{aligned}
& P^{\mu \nu}\left(\lambda_{p}\right) \equiv p^{\mu} e^{\nu}\left(\lambda_{p}\right)-e^{\mu}\left(\lambda_{p}\right) p^{\nu}, \\
& \hat{P}^{\mu \nu}\left(\lambda_{p}\right) \equiv e^{* \mu}\left(\lambda_{p}\right) p^{\nu}-p^{\mu} e^{* \nu}\left(\lambda_{p}\right)
\end{aligned}
$$

for the tensor $F^{\mu \nu}$ and

$$
\begin{aligned}
& \tilde{P}^{\mu \nu}\left(\lambda_{p}\right) \equiv \varepsilon^{\mu \nu \alpha \beta}\left(p_{\alpha} e_{\beta}\left(\lambda_{p}\right)-e_{\alpha}\left(\lambda_{p}\right) p_{\beta}\right) \\
& \hat{\tilde{P}}^{\mu \nu}\left(\lambda_{p}\right) \equiv \varepsilon^{\mu \nu \alpha \beta}\left(p_{\alpha} e_{\beta}^{*}\left(\lambda_{p}\right)-e_{\alpha}^{*}\left(\lambda_{p}\right) p_{\beta}\right)
\end{aligned}
$$

for the dual tensor $\tilde{F}^{\mu \nu}$.

Given the vector and tensor definitions above, the Lorentz-invariant scattering amplitude $\mathcal{M}_{\mathrm{S}}$ dedicated for scalar field exchange is expressed as (see eq. (A.33) in [27] for the detailed derivation)

$$
\mathcal{M}_{\mathrm{S}}=\frac{1}{4}\left(\frac{g}{M}\right)^{2} \frac{\left(P_{1} P_{2}\right)\left(\hat{P}_{3} \hat{P}_{4}\right)}{m^{2}-\left(p_{1}+p_{2}\right)^{2}}
$$

where the factors $\left(P_{i} P_{j}\right)$ in the numerator correspond to the vertex factors reflecting polarization states in the initial and final states, respectively. $(S T)$ is the abbreviation for a momentum-polarization tensor product such as $(S T) \equiv S_{\mu \nu} T^{\mu \nu}$ for four-momenta $s$ and $t$, that is, $\left(P_{1} P_{2}\right)$ corresponds to a momentum-tensor product for four-momenta $p_{1}$ and $p_{2}$. In the case of pseudoscalar exchange, we have only to replace the vertex factors with $\left(P_{1} \tilde{P}_{2}\right)\left(\hat{P}_{3} \hat{\tilde{P}}_{4}\right)$ using eq. (2.11). 
Hence, necessary momentum-polarization tensor products between four-momenta $s$ and $t$ with their polarization states $\lambda_{s}$ and $\lambda_{t}$ are summarized as

$$
\begin{aligned}
& S_{\mu \nu}\left(\lambda_{s}\right) T^{\mu \nu}\left(\lambda_{t}\right)=2\left\{(s \cdot t)\left(e\left(\lambda_{s}\right) \cdot e\left(\lambda_{t}\right)\right)-\left(s \cdot e\left(\lambda_{t}\right)\right)\left(t \cdot e\left(\lambda_{s}\right)\right)\right\}, \\
& \hat{S}_{\mu \nu}\left(\lambda_{s}\right) \hat{T}^{\mu \nu}\left(\lambda_{t}\right)=2\left\{(s \cdot t)\left(e^{*}\left(\lambda_{s}\right) \cdot e^{*}\left(\lambda_{t}\right)\right)-\left(s \cdot e^{*}\left(\lambda_{t}\right)\right)\left(t \cdot e^{*}\left(\lambda_{s}\right)\right)\right\}
\end{aligned}
$$

for scalar field exchange and

$$
\begin{aligned}
& S_{\mu \nu}\left(\lambda_{s}\right) \tilde{T}^{\mu \nu}\left(\lambda_{t}\right)=4 \varepsilon^{\mu \nu \alpha \beta} s_{\mu} e_{\nu}\left(\lambda_{s}\right) t_{\alpha} e_{\beta}\left(\lambda_{t}\right), \\
& \hat{S}_{\mu \nu}\left(\lambda_{s}\right) \hat{\tilde{T}}^{\mu \nu}\left(\lambda_{t}\right)=4 \varepsilon^{\mu \nu \alpha \beta} s_{\mu} e_{\nu}^{*}\left(\lambda_{s}\right) t_{\alpha} e_{\beta}^{*}\left(\lambda_{t}\right)
\end{aligned}
$$

for pseudoscalar exchange.

The actually used vertex factors for scalar and pseudoscalar field exchanges dedicated for this search with the fixed left-handed circular polarization state, $L$, of the inducing laser are expressed as follows:

$$
\begin{aligned}
& \text { scalar type }\left(P_{1}\left(l_{1}\right) P_{2}\left(l_{2}\right)\right)\left(\hat{P}_{3}\left(L_{3}\right) \hat{P}_{4}\left(L_{4}\right)\right) ; \\
& \text { pseudoscalar type }:\left(P_{1}\left(l_{1}\right) \tilde{P}_{2}\left(l_{2}\right)\right)\left(\hat{P}_{3}\left(L_{3}\right) \hat{\tilde{P}}_{4}\left(L_{4}\right)\right),
\end{aligned}
$$

where $l_{i}$ with $i=1,2$ represents mixing of linear polarization states $\{1\}$ and $\{2\}$ due to rotation of the $p_{1}-p_{2}$ reaction plane with respect to the linear polarization direction of the creation laser beam, while rotation of the $p_{3}-p_{4}$ reaction plane does not affect the circular polarization states of photons due to helicity conservation. We address only the $L$-state for $p_{3}\left(L_{3}\right)$ induced by the $L$-state for $p_{4}\left(L_{4}\right)$ in the inducing field. This is because vertex factors combining opposite circular polarization states always vanish, counter-intuitively, in both scalar and pseudoscalar exchanges based on eqs. (2.13) and (2.14).

\subsection{Outline of numerical calculations}

Figure 3 is a special example of an energy-incident-angle asymmetric collision that accidentally shares the common optical axis of the two laser beams, namely, the incident $p_{T}$ is set to zero. The actual procedures in the numerical calculation including fully asymmetric collisions are explained below based on the illustration in figure 4 . We first introduce momentum distributions $G_{p}$ as a function of polar angles $\Theta_{i}$ and azimuthal angles $\Phi_{i}$ mapped on the laboratory coordinates and energy distributions $G_{E}\left(\omega_{i}\right)$ for the creation (left, green) and inducing (right, red) lasers for individual photons $i=1,2,4$ by denoting $G$ representing normalized Gaussian distributions. Angular distributions in cylindrical coordinates $(\Theta, \Phi, z=0)$ (diameter corresponds to $\Theta$ ) are used here representing $G_{p}$ by fixing momentum norms at energies chosen from $G_{E}$. The concrete steps are then as follows. Step 0: select a finite-size segment of $p_{1}$ based on the $G_{E}\left(\omega_{1}\right) G_{p}\left(\Theta_{1}, \Phi_{1}\right)$ distributions. Step 1: a $z^{\prime}$-axis of zero- $p_{T}$ coordinates is defined by finding a paring $p_{2}$ that satisfies the resonance condition with respect to the selected $p_{1}$ and to a finite energy segment from $G_{E}\left(\omega_{2}\right)$ for a given mass parameter $m$. The kinematically possible ellipsoidal orbit is drawn with the purple belt on the angular distribution. Step 2: convert the polarization vectors 


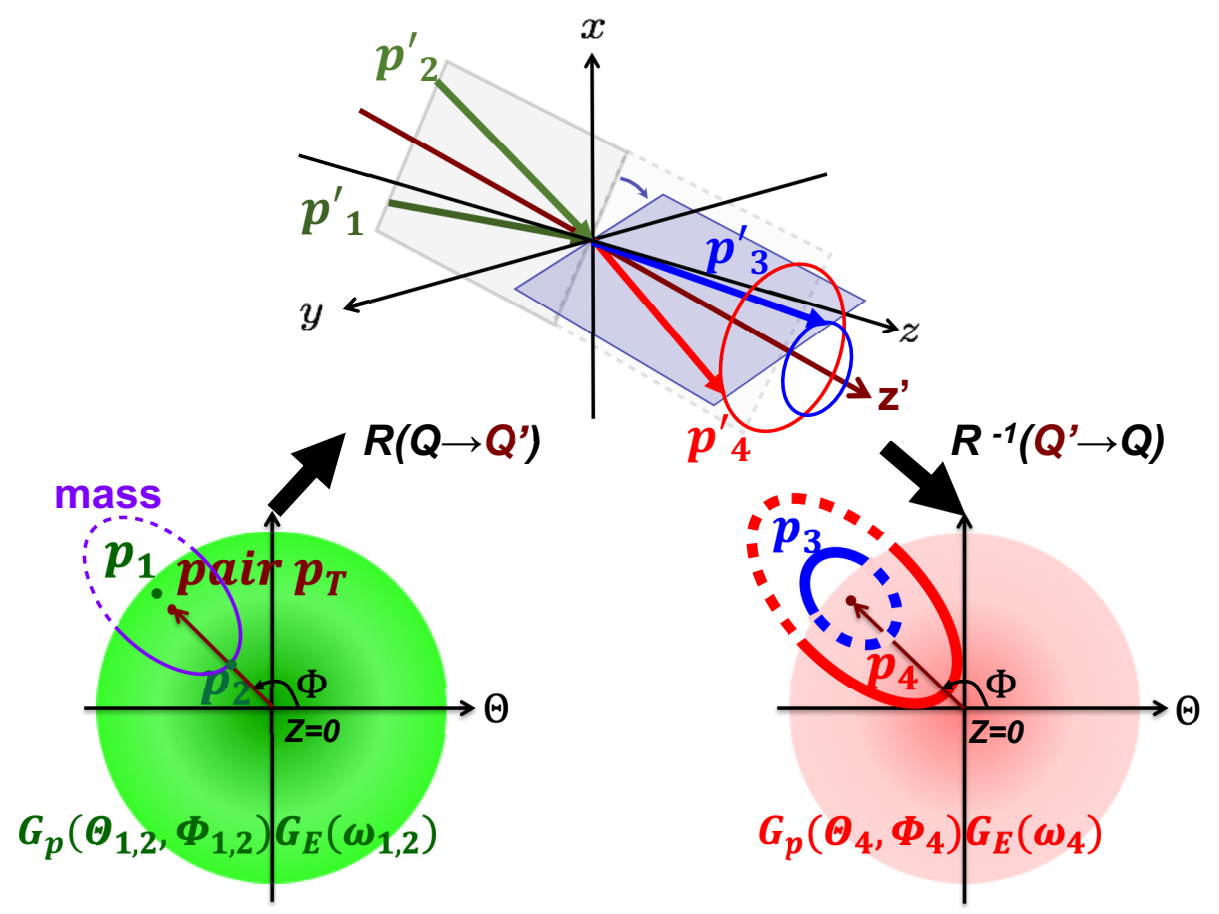

Figure 4. Flow of numerical calculations. The details are explained in the main text.

$e_{i}\left(\lambda_{i}\right)(i=1,2)$ from laboratory coordinates to zero- $p_{T}$ coordinates through coordinate rotation $R\left(Q \rightarrow Q^{\prime}\right)$. Step 3: calculate the invariant amplitude based on the vertex factors for scalar and pseudoscalar exchanges, respectively, in the given zero- $p_{T}$ coordinates where the axial symmetric nature of the final-state $p_{3}^{\prime}$ and $p_{4}^{\prime}$ around $z^{\prime}$ is preserved. Step 4: to evaluate the inducing effect with respect to $G_{E}\left(\omega_{4}\right) G_{p}\left(\Theta_{4}, \Phi_{4}\right)$ defined in laboratory coordinates, a matching fraction of $p_{4}$ is calculated after rotating back to the laboratory coordinates from the zero- $p_{T}$ coordinates, denoted by $R^{-1}\left(Q^{\prime} \rightarrow Q\right)$. Based on the spread of $G_{E}\left(\omega_{4}\right)$, the red ellipsoidal belt is determined via energy-momentum conservation. Note here that due to the circular polarization state of the inducing beam, any $p_{4}$ experiencing scattering can satisfy the polarization matching to the $p_{i}$ beam. Step 5: $p_{3}$ must balance with $p_{4}$ through energy-momentum conservation, so we can define a parametric signal energy spread via $\omega_{s} \equiv \omega_{3}=\omega_{1}+\omega_{2}-\omega_{4}$ as well as the polar and azimuthal angle spreads by taking the $G_{E}\left(\omega_{4}\right) G_{p}\left(\Theta_{4}, \Phi_{4}\right)$ distribution into account. The volume-wise interaction rate $\bar{\Sigma}_{\mathrm{I}}$ is integrated over the inducible solid angle of $p_{3}$ calculated from all the energy and angular spreads. Step 6: the signal yield $\mathcal{Y}_{c+i}$ is finally calculated based on eq. (2.3).

\section{$3 \quad$ Experimental setup}

Figure 5 illustrates the searching setup. A linearly polarized creation beam (Ti:sapphire pulsed laser) and a circularly polarized inducing beam (Nd:YAG pulsed laser) were combined with a dichroic mirror (DM2) in the transport chamber by sharing a common optical axis. At $\mathrm{P} 1$ in advance of the pulse compression, the linear polarization state of the creation laser was introduced. To transmit only P-polarized waves, P1 was made of 30 synthetic 


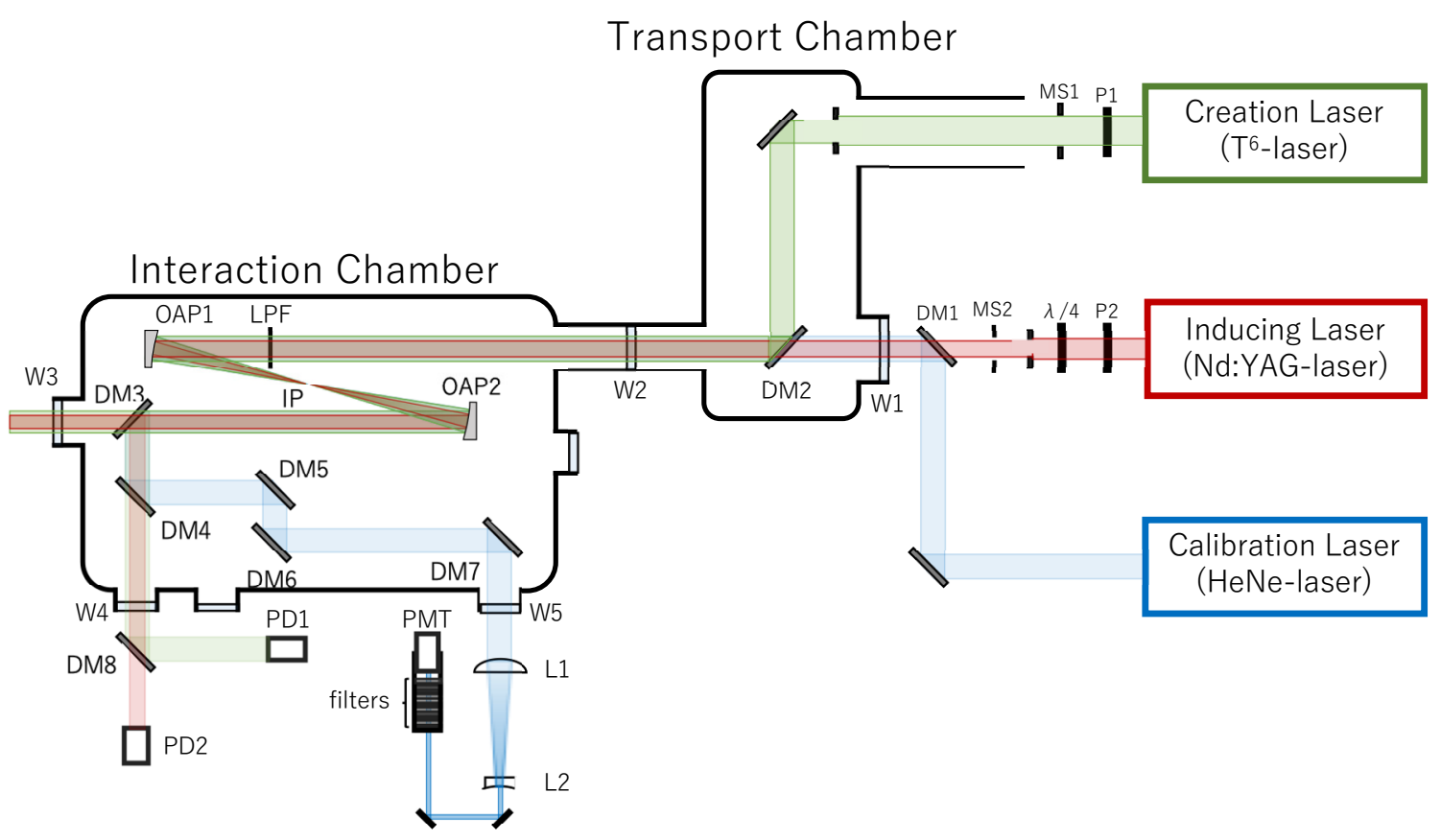

Figure 5. Schematic of the searching system contained in the beam transport chamber and interaction chamber designed to achieve $10^{-8} \mathrm{~Pa}$.

quartz plates tilted at Brewster's angle. The measured extinction ratio with the full energy shots was approximately 1000 (P-pol.) : 1 (S-pol.). The inducing laser was initially produced at $\mathrm{P} 2$ as a linearly polarized beam with a commercial polarization beam splitter with an extinction ratio of 200 (P-pol) : 1 (S-pol.), and then the linear polarized state was further converted into the circular polarization state by a quarter-plate $(\lambda / 4)$.

The central wavelengths of the creation and inducing lasers were 816 and $1064 \mathrm{~nm}$, respectively, their pulse durations were $31 \mathrm{fs}$ and $9 \mathrm{~ns}$, respectively, and their beam diameters were 37 and $16 \mathrm{~mm}$, respectively. Inside the interaction chamber, the two incident beams were focused simultaneously in the vacuum by an off-axis parabolic mirror (OAP1) with a focal length of $279.1 \mathrm{~mm}$. Based on an infinity-corrected optical system, focal-spot images of the two beams were transferred to a CCD camera outside the interaction chamber. The camera recorded the number of beam photons per pixel with a spatial resolution of $0.3 \mu \mathrm{m}$. A thin mesh with a known physical size located at the interaction point (IP) was used to calibrate the physical image size on the CCD camera. By adjusting optical components inside the transport chamber for the creation laser and ones outside the chamber for the inducing laser, spatial overlap was ensured based on the focal spot images of the two beams. The symmetrically placed identical off-axis parabolic mirror (OAP2) located at the point subtending the IP collected the signal waves by restoring the plane-wave propagation of the two incident beams. The intense incident beams were dumped through a dichroic mirror (DM3) that allowed the two incident beams to pass through while reflecting the signal waves.

With respect to the central wavelengths $\lambda_{c}$ and $\lambda_{i}$ for the creation and inducing lasers, respectively, the central wavelength $\lambda_{s}$ of the signal is defined by $\lambda_{s}=\left(2 / \lambda_{c}-1 / \lambda_{i}\right)^{-1}=$ $651 \mathrm{~nm}$. This wavelength is close to the $633 \mathrm{~nm}$ of a He:Ne laser, so a He:Ne laser was 
combined at DM1 and DM2 with the inducing and creation lasers, respectively, as a calibration source, by which one can trace the signal trajectory down to the signal detector. This calibration laser was used for aligning all the optical components inside the interaction chamber, and it also had a role in evaluating the acceptance factor from the IP to the detector with the specified circular polarization state.

The dichroic mirrors DM3-DM7 were custom-made and commonly reflected $651 \mathrm{~nm}$ with $99 \%$ while transmitting around $816 \mathrm{~nm}$ with $99 \%$ and $1064 \mathrm{~nm}$ with $95 \%$, to pick up the signal waves among the residual creation and inducing laser beams. To provide timing signals to synchronize the creation and inducing pulses and also to monitor the stability of the pulse energies, photodiodes (PD1, PD2) with a time resolution of $\sim 40$ ps were used by sampling the attenuated combined pulses that had passed through DM4.

A signal detector was used in the form of an R7400-01 single-photon-countable photomultiplier tube (PMT) manufactured by HAMAMATSU. The falling time resolution was $0.75 \mathrm{~ns}$, which is close to the waveform sampling resolution of $0.5 \mathrm{~ns}$ as explained below. For the signal wavelengths of $610-690 \mathrm{~nm}$ to be selected, a set of a low-pass filters transmitting above $610 \mathrm{~nm}$ and three types of band-pass filters transmitting $570-800 \mathrm{~nm}, 500-930 \mathrm{~nm}$, and $450-690 \mathrm{~nm}$ were installed in front of the PMT to remove residual photons from the intense incident lasers.

The voltages from the PMT and the two PDs as functions of time were recorded using a waveform digitizer with a time resolution of $0.5 \mathrm{~ns}$. The digitizer was triggered by a basic $10 \mathrm{~Hz}$ laser oscillator clock to which the incident timing between the creation and inducing lasers was synchronized. The incident rate of the creation laser was reduced to equi-interval $5 \mathrm{~Hz}$ by a mechanical shutter (MS1), whereas that of the inducing laser was adjusted to nonequi-interval $5 \mathrm{~Hz}$ by a mechanical shutter (MS2) to produce four staggered trigger patterns for the offline waveform analysis. The four types of trigger were as follows: (i) two-beam incidence, "S"; (ii) only inducing-laser incidence, "I"; (iii) only creation-laser incidence, "C"; (iv) no beam incidence, "P". These were issued in order over a data acquisition run, which ensured equal shot statistics per trigger pattern and also minimized the systematic uncertainties associated with subtractions between trigger patterns as explained in the next section.

\section{Data analysis}

\subsection{Counting number of photons by means of a peak finder}

The number of photons was evaluated based on the digitized waveform data from the PMT. In a waveform (sometimes referred to as a shot), voltage values $V_{i}$ were recorded with respect to individual sampling point $i$ within a $500 \mathrm{~ns}$ time interval as shown in figure 6 . Because the time resolution (i.e., the width of a time bin) is $0.5 \mathrm{~ns}, i$ runs from 1 to 1000. A peak finder identifies peak structures in a waveform on a shot-by-shot basis and counts the number of observed photons by the following steps: (i) the finder determines an average voltage $V_{0}$ over $i=1$ to 400; (ii) by setting a proper threshold voltage $V_{\text {th }}$, the finder identifies a minimum voltage $V_{m}$ at $i=m$ in the peak-like domain above $V_{\mathrm{th}}$; (iii) a half-voltage value is defined as $V_{\text {half }}=\left(V_{m}+V_{0}\right) / 2$; (iv) along the forward (f) and 


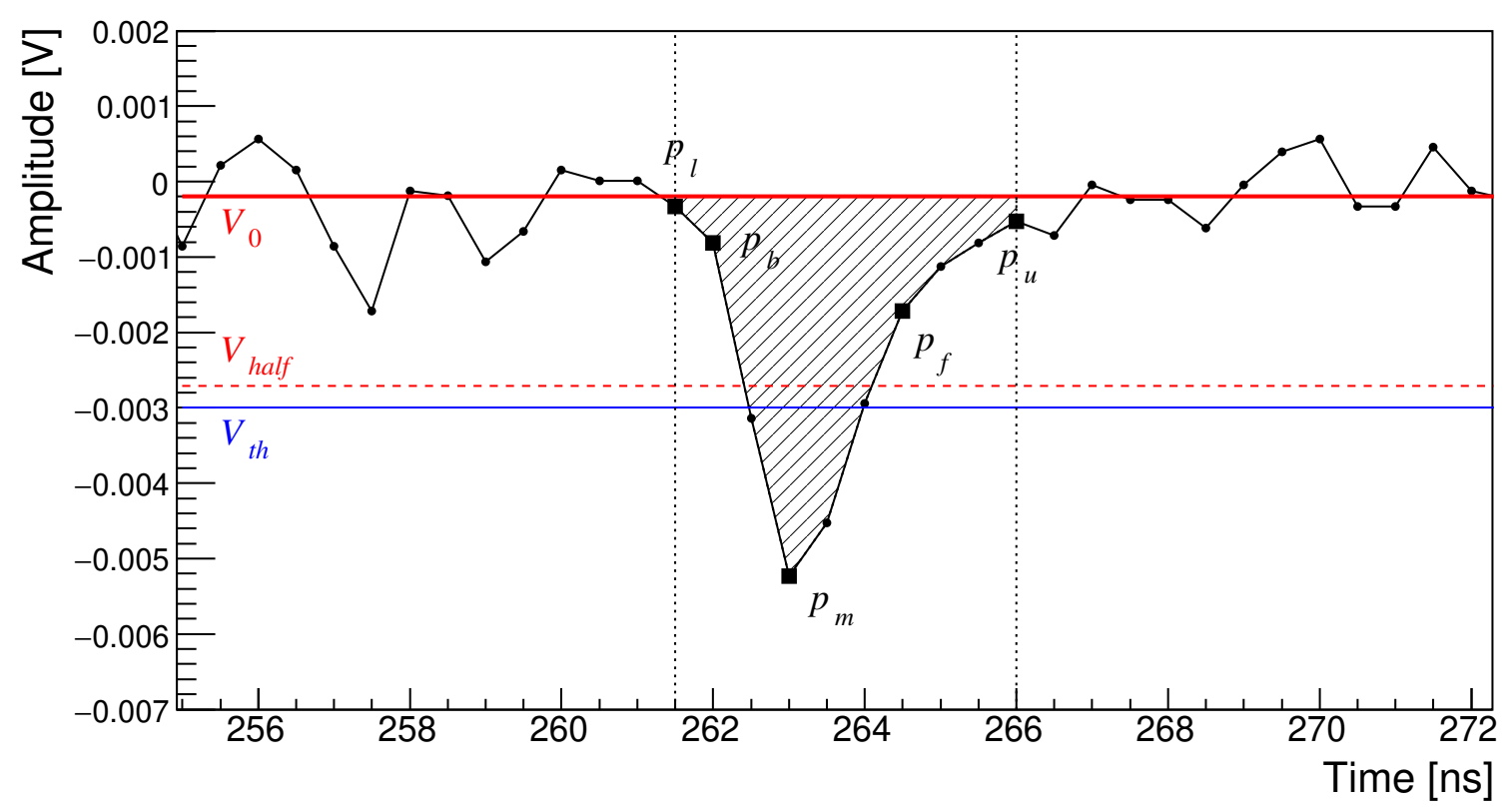

Figure 6. Waveform sample including a peak with a single trigger. The shaded area shows the integral range used to evaluate the charge sum of the peak structure.

backward (b) directions in time, the finder determines time points $i=f$ and $i=b$ at which voltage values exceed $V_{\text {half }}$; (v) from $i=f$, $b$, the falling $(i=l)$ and rising $(i=u)$ edges of a peak are defined as $l=m-(2(m-b)-1)$ and $u=m+2(f-m)$, respectively; (vi) a charge sum $Q$ contained in the peak-like structure depicted as the shaded area in figure 6 is evaluated as $Q=\sum_{i=l}^{u} V_{i} \Delta t / R$ with $\Delta t=0.5 \mathrm{~ns}$ and $R=50 \Omega$; (vii) given a single-photon equivalent charge $Q_{\text {p.e. }}=(8.206 \pm 0.015) \times 10^{-14} \mathrm{C}$ calibrated in advance, $Q / Q_{\text {p.e. }}$ provides the observed number of photons in the peak-like structure.

\subsection{Pressure dependence of atomic four-wave mixing process}

Nonlinear optical parametric effects caused by third-order polarization susceptibility $\chi^{(3)}$, so-called four-wave mixing (FWM) [31], are expected to occur even in the residual gas in the interaction vacuum chamber. This can be a dominant background source because the wavelengths generated in atomic FWM are nearly equal to those of stimulated scattering in a vacuum due to the kinematic similarity based on energy-momentum conservation between the initial and final state photons. Therefore, we can refer to the searched-for stimulated scattering process as FWM in a vacuum. Meanwhile, atomic FWM is quite useful for ensuring spatiotemporal synchronization between the creation and inducing pulse lasers in QPS. To validate our searching system, we measured the pressure dependence of the number of atomic FWM photons. Because the peak finder can provide the falling edges of photon incident peaks in the waveforms, the arrival-time distribution as shown in figure 7 is measurable in units of the number of photons for each of the four trigger patterns. This figure shows the results measured at $10 \mathrm{~Pa}$. The peak structures appeared in trigger patterns $\mathrm{S}$ and $\mathrm{C}$. The peak seen in pattern $\mathrm{C}$ is expected because of plasma creation at the IP because the creation laser intensity is high enough to induce ionization of residual atoms. 

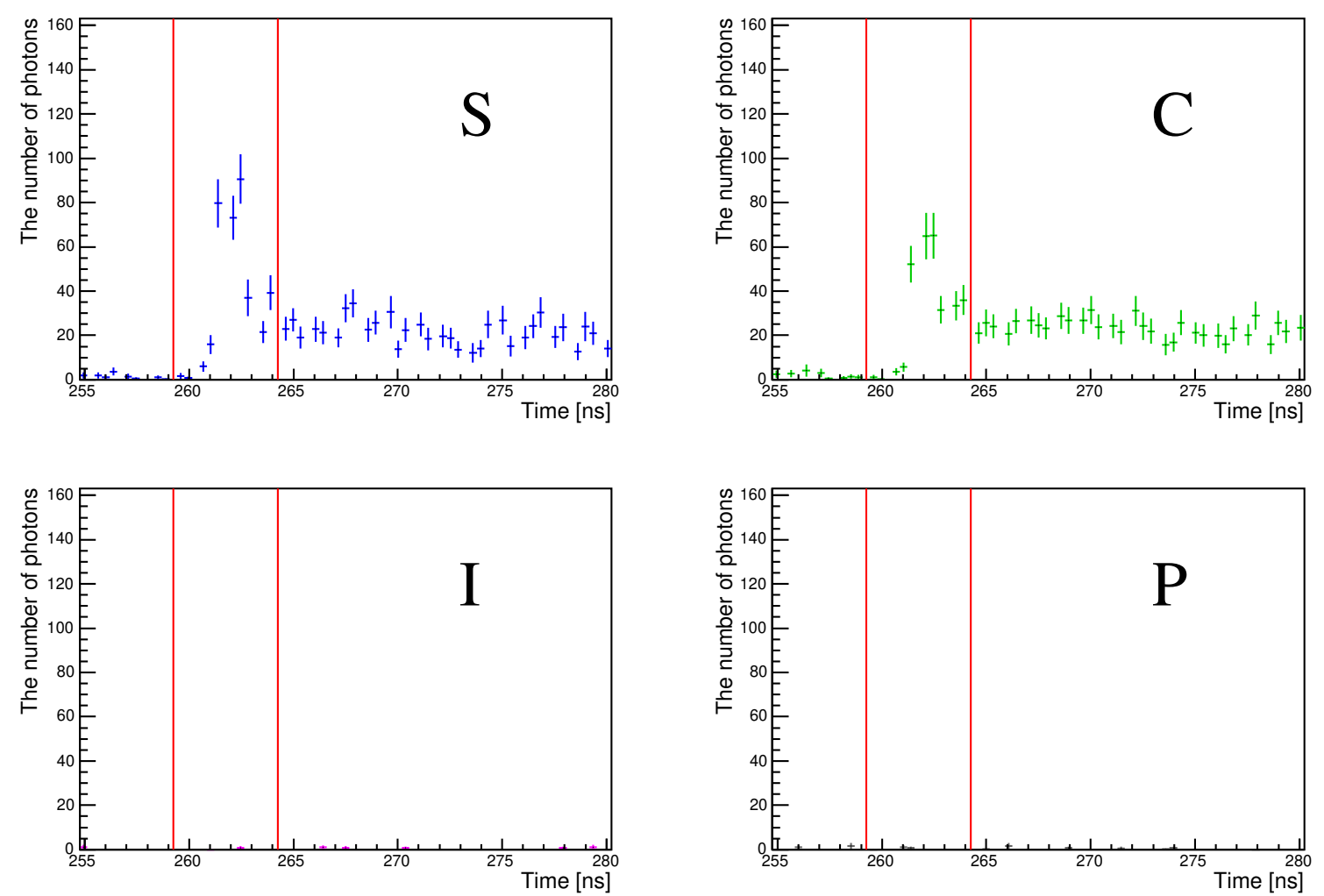

Figure 7. Arrival-time distributions of number of observed photons by combining the Ppolarization state (creation) and the left-handed circular polarization state (inducing) for individual trigger patterns at $10 \mathrm{~Pa}$. The individual time windows in which signal photons are expected to be detected are indicated with the two red lines.

In contrast, the intensity of the inducing laser field is much lower because of the long time duration, as seen in pattern I where no peak is found. Meanwhile, the higher peak seen in pattern $\mathrm{S}$ is expected to be the sum between the atomic FWM and the plasma-origin photon yields.

The basic assumption that addition of the number of photons in individual trigger patterns corresponds to the number of photons in trigger pattern $\mathrm{S}$ is indeed supported by the following subtraction analysis. The acceptance-uncorrected number of atomic FWM photons, $N_{\mathrm{S}}$, can be obtained via

$$
N_{\mathrm{S}}=\left(n_{\mathrm{S}}-n_{\mathrm{P}}\right)-\left(n_{\mathrm{C}}-n_{\mathrm{P}}\right)-\left(n_{\mathrm{I}}-n_{\mathrm{P}}\right)=n_{\mathrm{S}}-n_{\mathrm{C}}-n_{\mathrm{I}}+n_{\mathrm{P}},
$$

where $n_{i}$ is the number of photons for trigger pattern $i$ measured in the time interval subtended by the two red vertical lines corresponding to the signal generation timing window. Figure 8 shows the pressure dependence of the number of signal photons per shot, which is expected to be dependent upon the square of the pressure because the photon yield of the atomic FWM should be proportional to $\left(\chi^{(3)}\right)^{2} \propto(\text { density })^{2} \propto$ (pressure $)^{2}$. The dependence was thus fitted with

$$
N_{\mathrm{S}} / \operatorname{shot}=a \mathcal{P}^{b},
$$




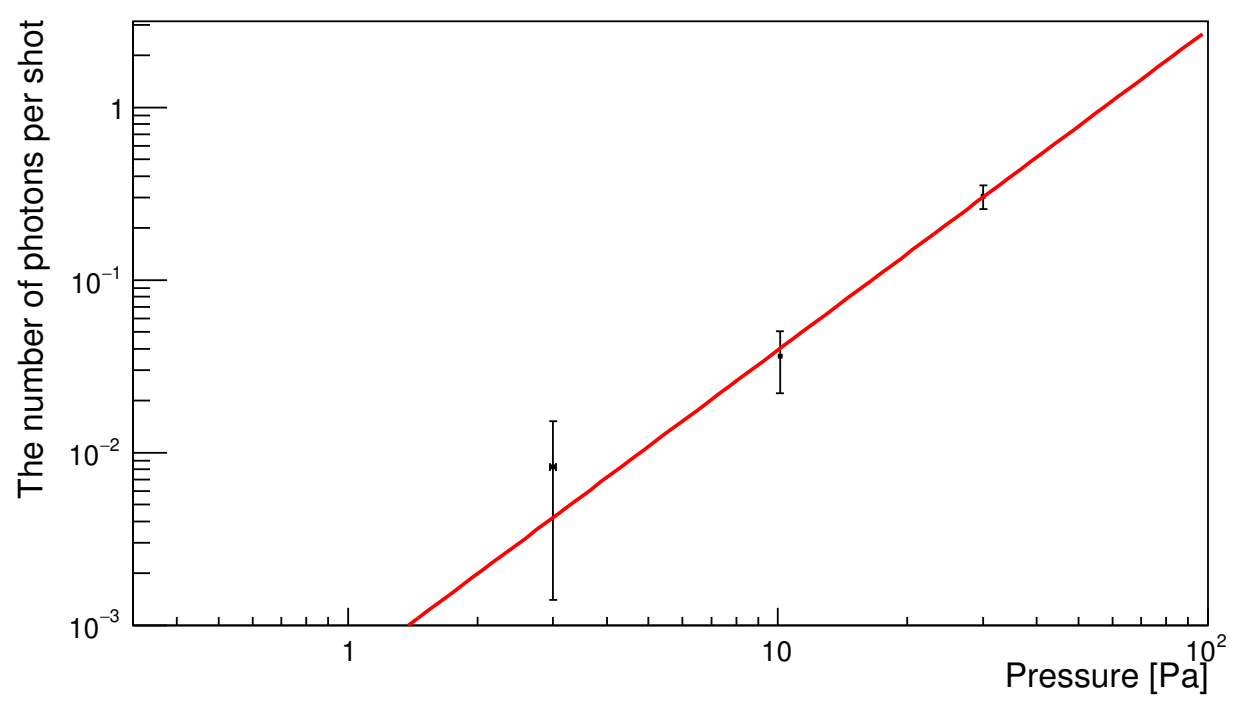

Figure 8. Pressure dependence of number of four-wave-mixing photons per shot from residual atoms inside interaction chamber when P-pol. (creation) and left-handed circular (inducing) polarization laser pulses are combined and focused.

where $a$ and $b$ are fitting parameters and $\mathcal{P}$ is pressure. The error bars are the quadratic sum of the statistical error propagation associated with the subtraction process between trigger patterns and systematic uncertainties of focal-point stability during a run period. We explain these uncertainties in the following subsection. As expected, $b=1.85 \pm 0.35$ is close to the expected behavior $N_{\mathrm{S}} \propto \mathcal{P}^{2}$ in atomic physics [29,31]. Note that this pressure dependence itself is valuable as data because the special combination between linear and circular polarization state beams is a very rare case in atomic physics.

\subsection{Focal-point stability}

The systematic uncertainties due to focal-point fluctuations were estimated from overlaps between the two laser focal-spot profiles measured by the common CCD camera sensitive to both wavelengths. Figure 9 shows typical focal-spot images of the creation and inducing lasers.

With the local intensity per CCD pixel of the monitor camera, $N(x, y)$, the overlap factor $O$ is defined as

$$
O \equiv \sum_{x}^{c} \sum_{y}^{c} N_{c}^{2}(x, y) N_{i}(x, y)
$$

where the subscripts $c$ and $i$ specify the creation and inducing lasers, respectively. The summations were taken over the area framed by the full width at half maximum of the creation laser intensity profile. Fluctuations of the overlap factors with respect to the mean overlap factor $\left(O_{\mathrm{I}}+O_{\mathrm{F}}\right) / 2$ were then evaluated as

$$
\delta N_{\mathrm{S}}=\left|N_{\mathrm{S}} \frac{O_{\mathrm{I}}-O_{\mathrm{F}}}{O_{\mathrm{I}}+O_{\mathrm{F}}}\right|,
$$

where $O_{\mathrm{I}, \mathrm{F}}$ are the overlap factors at the beginning and end, respectively, of a $2000 \mathrm{~s}$ unit run period. 

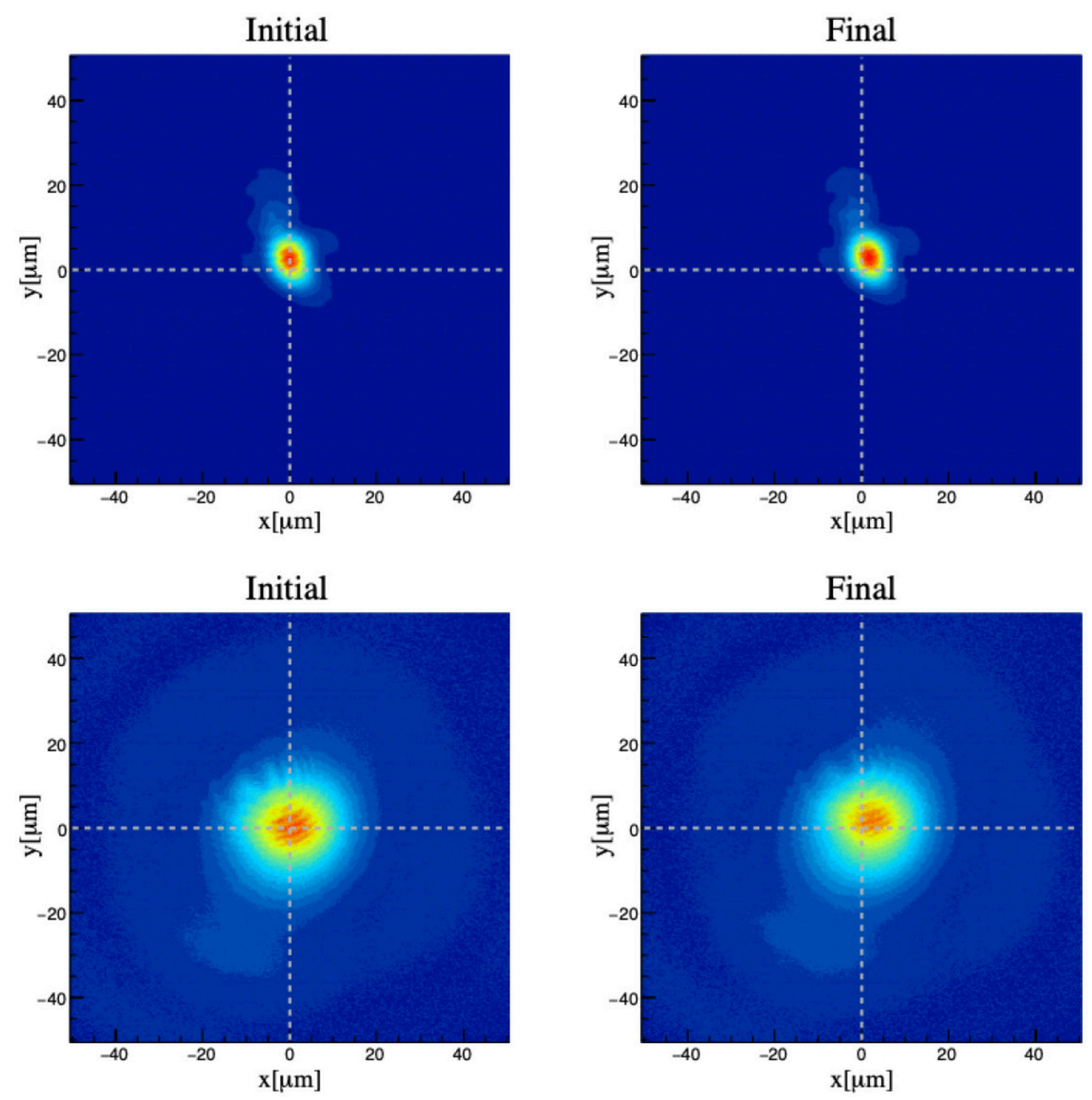

Figure 9. Beam profiles of creation (upper) and inducing (lower) lasers at interaction point as captured by a common CCD camera. The left and right figures correspond to typical images taken at the beginning and end of a unit run period, where slight deviations in the focal spots are seen.

\subsection{Effective energy fraction in Gaussian beams}

Figure 10 shows the results of fitting the focal-plane intensity profiles of the creation and inducing beams with two-dimensional Gaussian distributions constrained by $x-y$ symmetry. From the fitting results $\sigma_{x y}=7$ and $17 \mu \mathrm{m}$ for the creation and inducing lasers, respectively, we evaluated the effective energy fraction contained in the region within $3 \sigma_{x y}$ among the entire intensity profiles, including the peripheral diffraction parts that are assumed not to contribute to stimulated photon-photon scattering.

\section{Search result}

By combining linearly polarized creation laser pulses and circularly polarized inducing laser pulses, searches for scalar and pseudoscalar resonance states were performed at a vacuum 

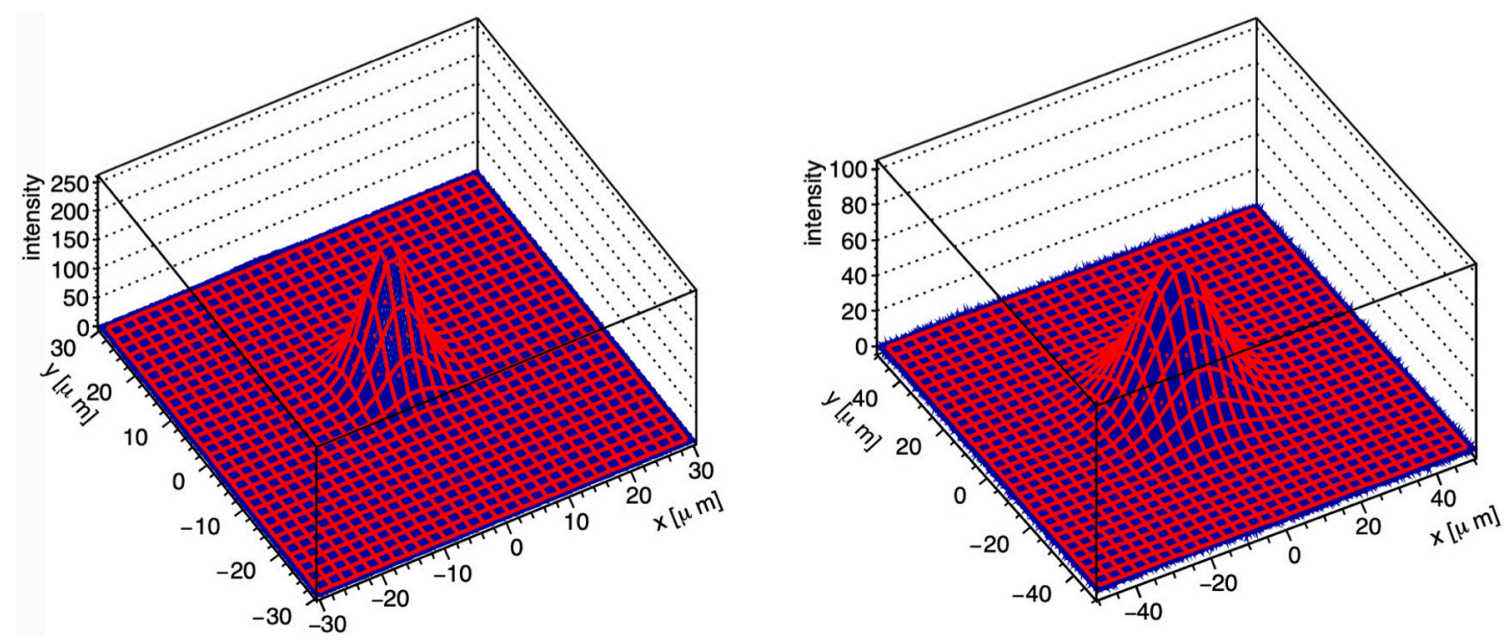

Figure 10. Fitting of focal-plane intensity profiles with two-dimensional Gaussian distributions constrained by $x-y$ symmetry for creation (left) and inducing (right) beams.

pressure of $2.6 \times 10^{-5} \mathrm{~Pa}$. The arrival-time distributions of photons identified by the peak finder are shown in figure 11 for the individual trigger patterns. The area subtended by the two red lines corresponds to the expected signal timing window. The total number of shots in trigger pattern $\mathrm{S}$ was $W_{\mathrm{S}} \equiv 2.9993 \times 10^{4}$.

Figure 12 shows the arrival-time distribution of the number of photons after subtraction between different trigger patterns based on eq. (4.1). The total number of signal photons within the signal incident timing window was obtained as

$$
N_{\mathrm{S}}=4.9 \pm 22.8 \text { (stat.) } \pm 22.8 \text { (syst. I) } \pm 3.8 \text { (syst. II) } \pm 3.7 \text { (syst. III) }
$$

Systematic error I was estimated by measuring the root-mean-square of the number of photon-like signals excluding the signal window, corresponding to the baseline uncertainty on the number of photons. Systematic error II reflects time variations of the overlap factors defined in eq. (4.4) between the focal spots of the creation and inducing lasers. Note that this equation contains fluctuations of beam energies during a run period as well as the pointing fluctuations. Systematic error III was obtained by changing the threshold value in the peak finder, $V_{\mathrm{th}}=(-1.3 \pm 0.1) \mathrm{mV}$, by assuming a uniform distribution from -1.2 to $-1.4 \mathrm{mV}$.

\section{Upper limits on coupling-mass relation for ALP exchanges}

From the result in (5.1), we conclude that no signal photons in the quasi-vacuum state were observed based on the total uncertainty. Indeed, this result is also consistent with the expected number of background photons per shot (efficiency-uncorrected) due to residual gases, estimated as

$$
N_{\text {gas }} / \text { shot }=1.8 \times 10^{-12} \text { photons }
$$

by extrapolating to $2.6 \times 10^{-5}$ with eq. (4.2). In addition, for the given total statistics, the expectation value based on the QED photon-photon scattering process, which is the 

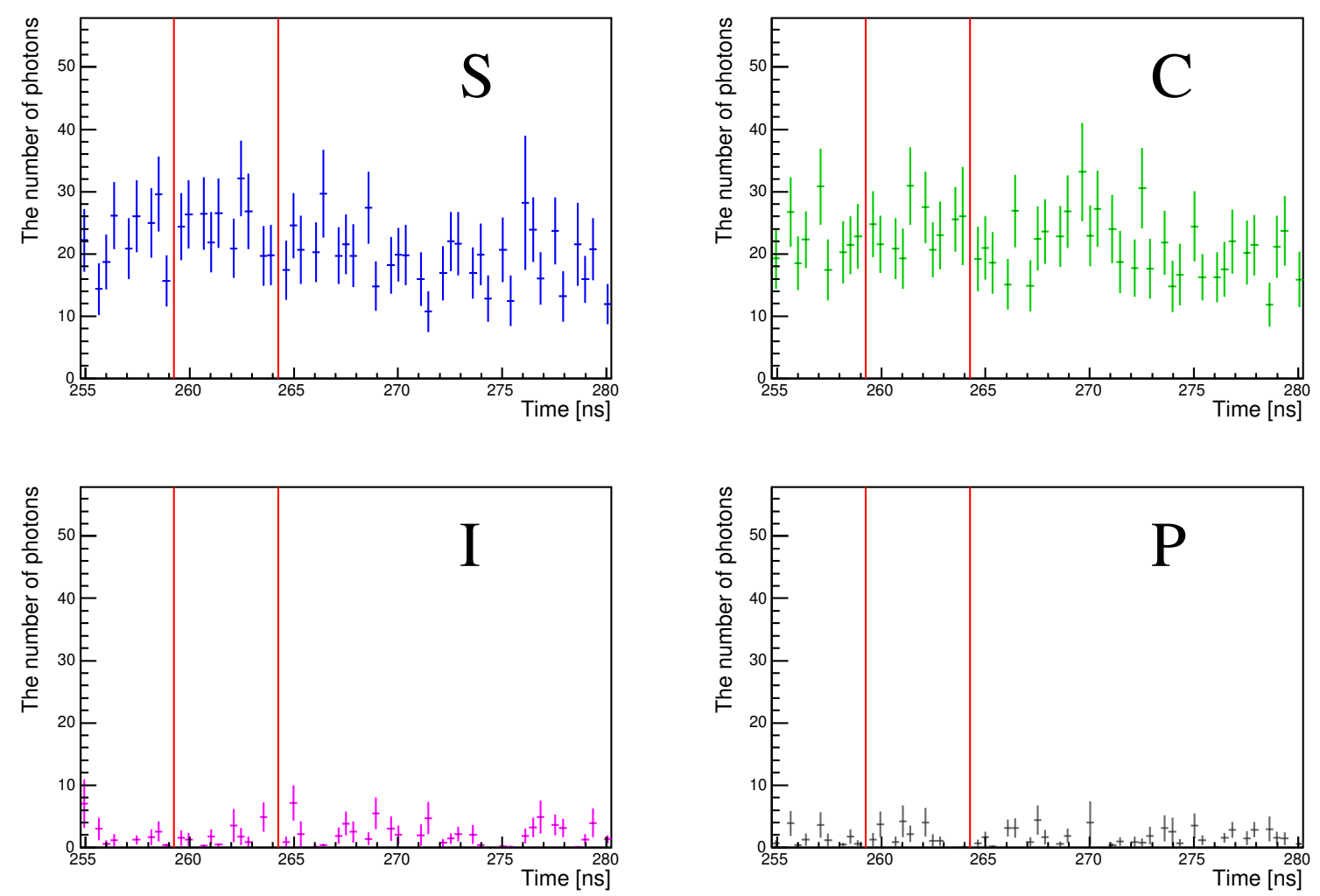

Figure 11. Arrival-time distributions of detected photons at $2.6 \times 10^{-5} \mathrm{~Pa}$ for trigger patterns $\mathrm{S}, \mathrm{C}$, $\mathrm{I}$, and $\mathrm{P}$ by combining the P-polarization state (creation) and the left-handed circular polarization state (inducing).

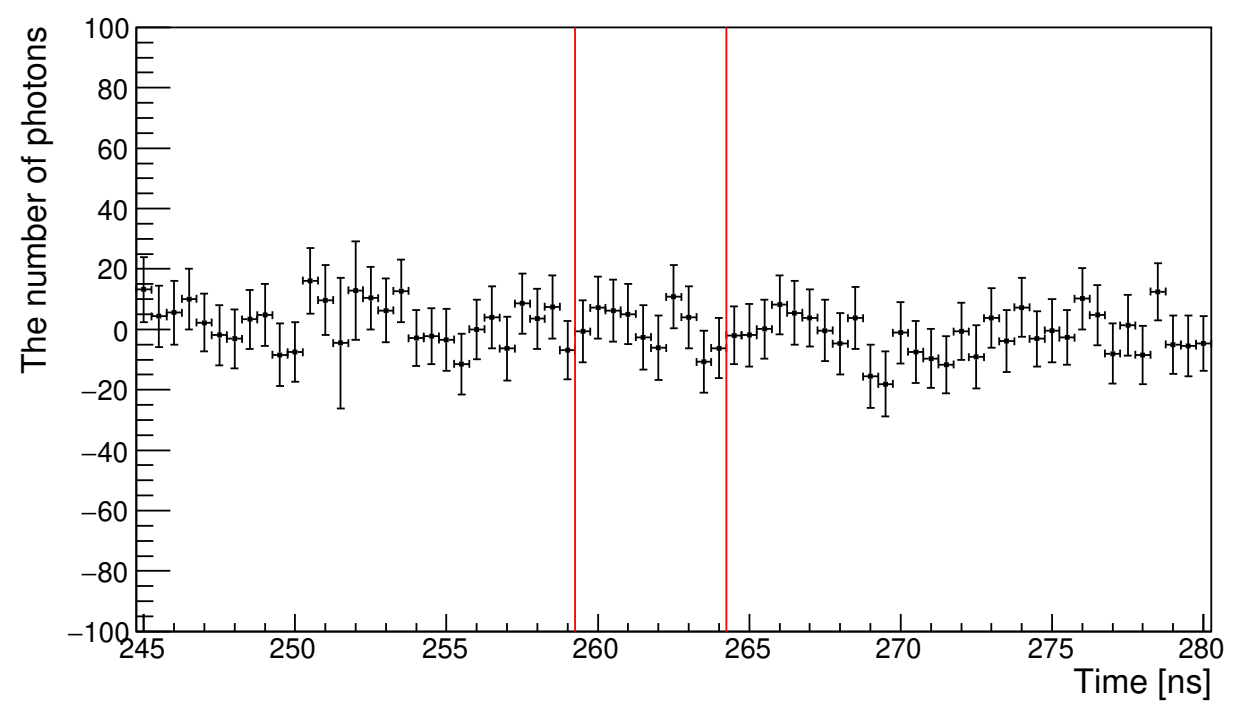

Figure 12. Arrival-time distribution of number of photons obtained by applying eq. (4.1) to the entire timing windows including the signal window enclosed by the two red lines at $2.6 \times 10^{-5} \mathrm{~Pa}$ by combining the P-polarization state (creation) and the left-handed circular polarization state (inducing). 


\begin{tabular}{lr}
\hline Parameter & Value \\
\hline Centeral wavelength of creation laser $\lambda_{c}$ & $816 \mathrm{~nm}$ \\
Relative linewidth of creation laser, $\delta \omega_{c} /\left\langle\omega_{c}\right\rangle$ & $1.2 \times 10^{-2}$ \\
Duration time of creation laser, $\tau_{c}$ & 31 fs $(=36 \mathrm{fs}(\mathrm{FWHM}) / \sqrt{2 \ln 2})$ \\
Measured creation laser energy per $\tau_{c}, E_{c}$ & $(100 \pm 5) \mu \mathrm{J}$ \\
Creation energy fraction within $3 \sigma_{x y}$ focal spot, $f_{c}$ & 0.85 \\
Effective creation energy per $\tau_{c}$ within $3 \sigma_{x y}$ focal spot & $E_{c} f_{c}=85 \mu \mathrm{J}$ \\
Effective number of creation photons, $N_{c}$ & $3.5 \times 10^{14}$ photons \\
Beam diameter of creation laser beam, $d_{c}$ & $(37.0 \pm 0.8) \mathrm{mm}$ \\
Polarization & linear $(\mathrm{P}-$ polarized state $)$ \\
\hline Central wavelength of inducing laser, $\lambda_{i}$ & $1064 \mathrm{~nm}$ \\
Relative linewidth of inducing laser, $\delta \omega_{i} /\left\langle\omega_{i}\right\rangle$ & $1.0 \times 10^{-4}$ \\
Duration time of inducing laser beam, $\tau_{\mathrm{ibeam}}$ & $9 \mathrm{~ns}$ \\
Measured inducing laser energy per $\tau_{\mathrm{ibeam}}, E_{i}$ & $(200 \pm 4) \mu \mathrm{J}$ \\
Linewidth-based duration time of inducing laser, $\tau_{i} / 2$ & $\hbar /\left(2 \delta \omega_{i}\right)=2.8 \mathrm{ps}$ \\
Inducing energy fraction within $3 \sigma_{x y}$ focal spot, $f_{i}$ & 0.87 \\
Effective inducing energy per $\tau_{i}$ within $3 \sigma_{x y}$ focal spot & $E_{i}\left(\tau_{i} / \tau_{\text {ibeam }}\right) f_{i}=0.11 \mu \mathrm{J}$ \\
Effective number of inducing photons, $N_{i}$ & $5.9 \times 10^{11}$ photons \\
Beam diameter of inducing laser beam, $d_{i}$ & $(15.8 \pm 0.3) \mathrm{mm}$ \\
Polarization & circular $($ left-handed state $)$ \\
\hline Focal length of off-axis parabolic mirror, $f$ & $279.1 \mathrm{~mm}$ \\
Single-photon detection efficiency, $\epsilon_{d}$ & $1.4 \%$ \\
Efficiency of optical path from IP to PMT, $\epsilon_{L}$ & $33 \%$ \\
\hline Total number of shots in trigger pattern S, $W_{\mathrm{S}}$ & 32.7 \\
$\delta N_{\mathrm{S}}$ & $2.9993 \times 10^{4}$ shots \\
\hline
\end{tabular}

Table 1. Experimental parameters used to numerically calculate the upper limits on the couplingmass relations. The effective numbers of photons, $N_{c}$ and $N_{i}$, were used for the limit calculations.

only possible process in the standard model, is negligibly low at $E_{\mathrm{cms}}<1 \mathrm{eV}[28]$ even though the stimulation effect is taken into account [32]. Therefore, with respect to a null hypothesis following a Gaussian distribution, we provide the upper limits on the couplingmass relation by assuming scalar and pseudoscalar field exchanges with the experimental parameters in table 1.

We note that the pulse duration of the Nd:YAG laser, $\tau_{\text {ibeam }}$, in table 1 is not corresponding to that of the Fourier transform limit due to the different scheme to generate pluses from that of Ti:sapphire laser in which time duration close to reaching the Fourier transform limit is obtained. Thus, the effective time duration reaching the Fourier transform limit, $\tau_{i}$, which can overlap with the creation pulse duration, $\tau_{c}$, is evaluated from the spectrum linewidth of the Nd:YAG laser. This treatment is consistent with the basic assumption in [27] where the inducing effect is evaluated based on overlapping pulses individually reaching Fourier transform limits. In addition to the effective time durations, by considering the spatially overlapping regions within $3 \sigma_{x y}$ focal spots which are consistent with the Gaussian shapes, the effective numbers of photons per pulses, $N_{c}$ and $N_{i}$, were 
used for the following limit calculations. In this sense, the following results correspond to conservative upper limits, because the effective beam energies stored in pulses are very much reduced.

As for the upper mass range, because of the inclusion of general asymmetric collisions, this search is sensitive to a heavier mass range compared with the symmetric collision range, expressed as

$$
m=2 \omega_{c} \sin \Delta \theta \sim 2 \omega_{c} \frac{d_{c}}{2 f}=0.21 \mathrm{eV}
$$

based on values in table 1 with $\Delta \theta \equiv d /(2 f)$ defined by the focal length $f$ and beam diameter $d$ of the creation laser in figure 1 . Note, however, that this value is merely a reference mass at which the maximum sensitivity is expected.

A confidence level $1-\alpha$ to exclude a null hypothesis is expressed as

$$
1-\alpha=\frac{1}{\sqrt{2 \pi} \sigma} \int_{\mu-\delta}^{\mu+\delta} e^{-(x-\mu)^{2} /\left(2 \sigma^{2}\right)} d x=\operatorname{erf}\left(\frac{\delta}{\sqrt{2} \sigma}\right),
$$

where $\mu$ is the expected value of an estimator $x$ following a hypothesis, and $\sigma$ is one standard deviation. In this search, the estimator $x$ corresponds to $N_{\mathrm{S}}$, and we assign the acceptance-uncorrected uncertainty $\delta N_{\mathrm{S}}$ from the quadratic sum of all error components in the result (5.1) as the one standard deviation $\sigma$ around the mean value $\mu=0$. In this search, the null hypothesis is produced from fluctuations of the number of photonlike signals following a Gaussian distribution whose expectation value, $\mu$, is zero for the given total number of shots, $W_{\mathrm{S}}=2.9993 \times 10^{4}$. This is because $N_{\mathrm{S}}$ is calculated from subtractions between different trigger patterns whose baseline fluctuations, in principle, should follow Gaussian distributions individually. To obtain a confidence level of $95 \%$, $2 \alpha=0.05$ with $\delta=2.24 \sigma$ is used, where a one-sided upper limit by excluding above $x+\delta,{ }^{1}$ is applied. To evaluate the upper limits on the coupling-mass relation, we then solved

$$
2.24 \delta N_{\mathrm{S}}=\mathcal{Y}_{c+i}(m, g / M ; P) t_{a} r \epsilon
$$

numerically based on eq. (2.2) with respect to $m$ and $g / M$ for a set of experimental parameters $P$ in table 1 , where $t_{a} r=W_{\mathrm{S}}=2.9993 \times 10^{4}$ and the overall efficiency $\epsilon \equiv \epsilon_{\mathrm{opt}} \epsilon_{d}$ with the optical path acceptance $\epsilon_{\mathrm{opt}}$ to the $p_{3}$ detector position and the single $p_{3}$-photon detection efficiency $\epsilon_{d}$ were used. Figures 13 and 14 show the obtained upper limits on the coupling-mass relations for scalar and pseudoscalar fields, respectively, at a $95 \%$ confidence level. Note that based on eq. (2.15), we used $\epsilon_{\mathrm{opt}}=\epsilon_{L}$ as the optical path acceptance factor, where $\epsilon_{L}$ is the acceptance factor with respect to left-handed circularly polarized photons measured from the IP to the $p_{3}$-detection position. This is because both scalar and pseudoscalar fields can couple only to the same helicity state as that of the inducing field, which is provided as the left-handed state in the searching setup.

\section{Conclusions}

By combining linearly polarized creation laser pulses and circularly polarized inducing laser pulses, we have searched for scalar and pseudoscalar fields via stimulated resonant

\footnotetext{
${ }^{1}$ See eq. (36.56) in [33].
} 


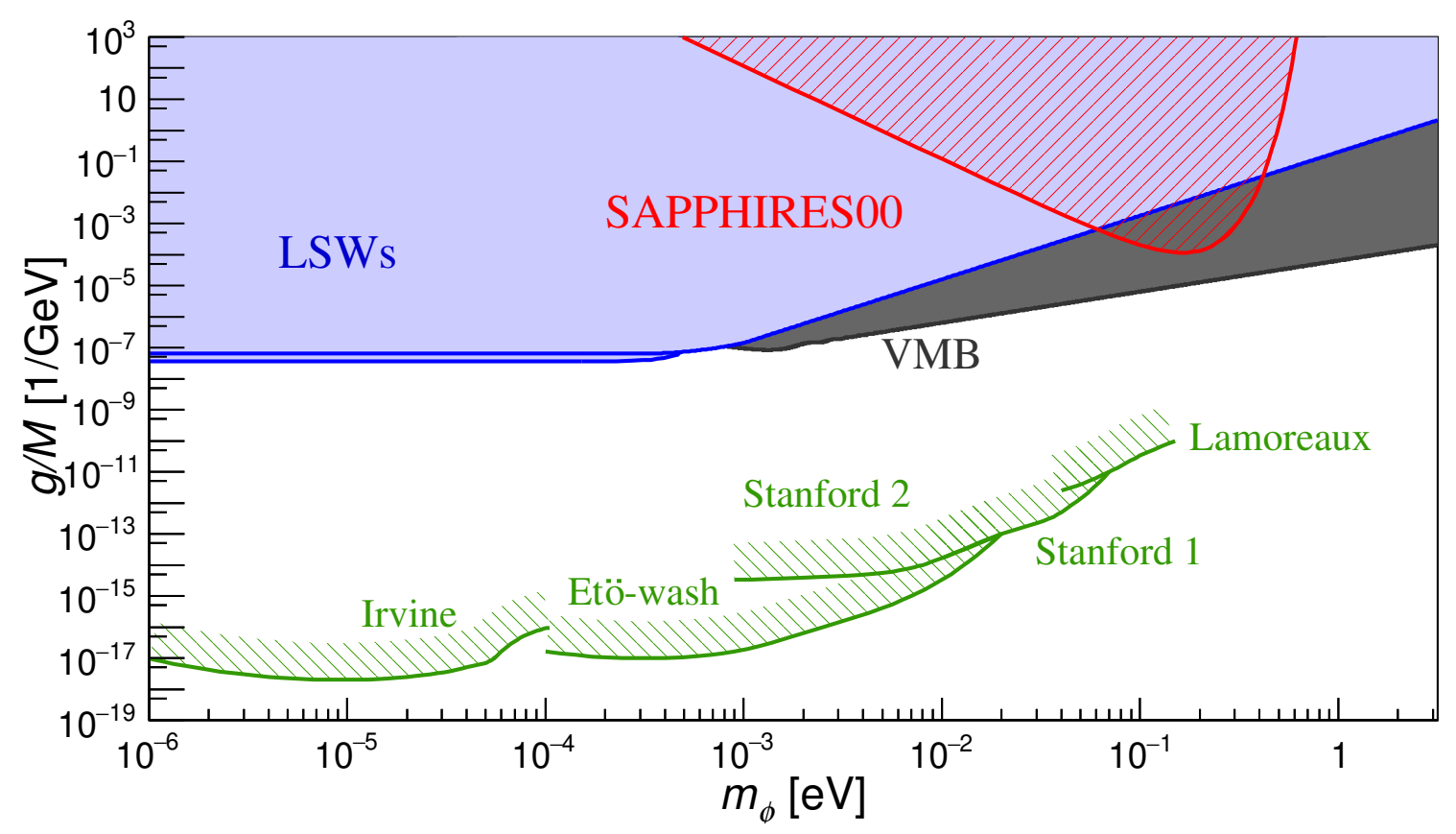

Figure 13. Upper limits on coupling-mass relation for scalar field $(\phi)$ exchanges. The red shaded area labeled "SAPPHIRES00" is the region excluded by this work (stimulated resonant scattering). The light-blue area is the region for scalar fields excluded by the "Light Shining through a Wall experiments (LSWs)" (OSQAR [34] and ALPS [35]) with simplification of the sine-function part to unity above $10^{-3} \mathrm{eV}$ for drawing convenience. The gray area is the result from the "Vacuum Magnetic Birefringence (VMB)" experiment (PVLAS [36]). The green shaded areas are regions excluded based on non-Newtonian force searches ("Irvine" [37], "Eto-wash" [38, 39], "Stanford1" [40], "Stanford2" [41]) and on Casimir force measurements ("Lamoreaux" [42]).

scattering by focusing two-color pulsed lasers: $0.10 \mathrm{~mJ} / 31 \mathrm{fs}$ at $816 \mathrm{~nm}$ and $0.20 \mathrm{~mJ} / 9 \mathrm{~ns}$ at $1064 \mathrm{~nm}$. The observed number of signal photons in the quasi-vacuum state at $2.6 \times 10^{-5} \mathrm{~Pa}$ was $4.9 \pm 22.8$ (stat.) \pm 22.8 (syst. I) \pm 3.8 (syst. II) \pm 3.7 (syst. III). We thus conclude that no significant signal was observed in this search. The expected number of signal photons from the residual gas is sufficiently low based on the upper limit from the measurement of the pressure dependence. Based on the assumption that uncertainties are dominated by systematic fluctuations around the zero expectation value following a Gaussian distribution and the fully asymmetric collisional geometry in quasi-parallel stimulated photon-photon scattering, we provided upper limits on the coupling-mass relations for scalar and pseudoscalar fields at a 95\% confidence level in the sub-eV mass range.

\section{Acknowledgments}

K. Homma acknowledges the support of the Collaborative Research Program of the Institute for Chemical Research of Kyoto University (Grant Nos. 2018-83, 2019-72, 202085, and 2021-88) and Grants-in-Aid for Scientific Research Nos. 17H02897, 18H04354, 19K21880, and 21H04474 from the Ministry of Education, Culture, Sports, Science and 


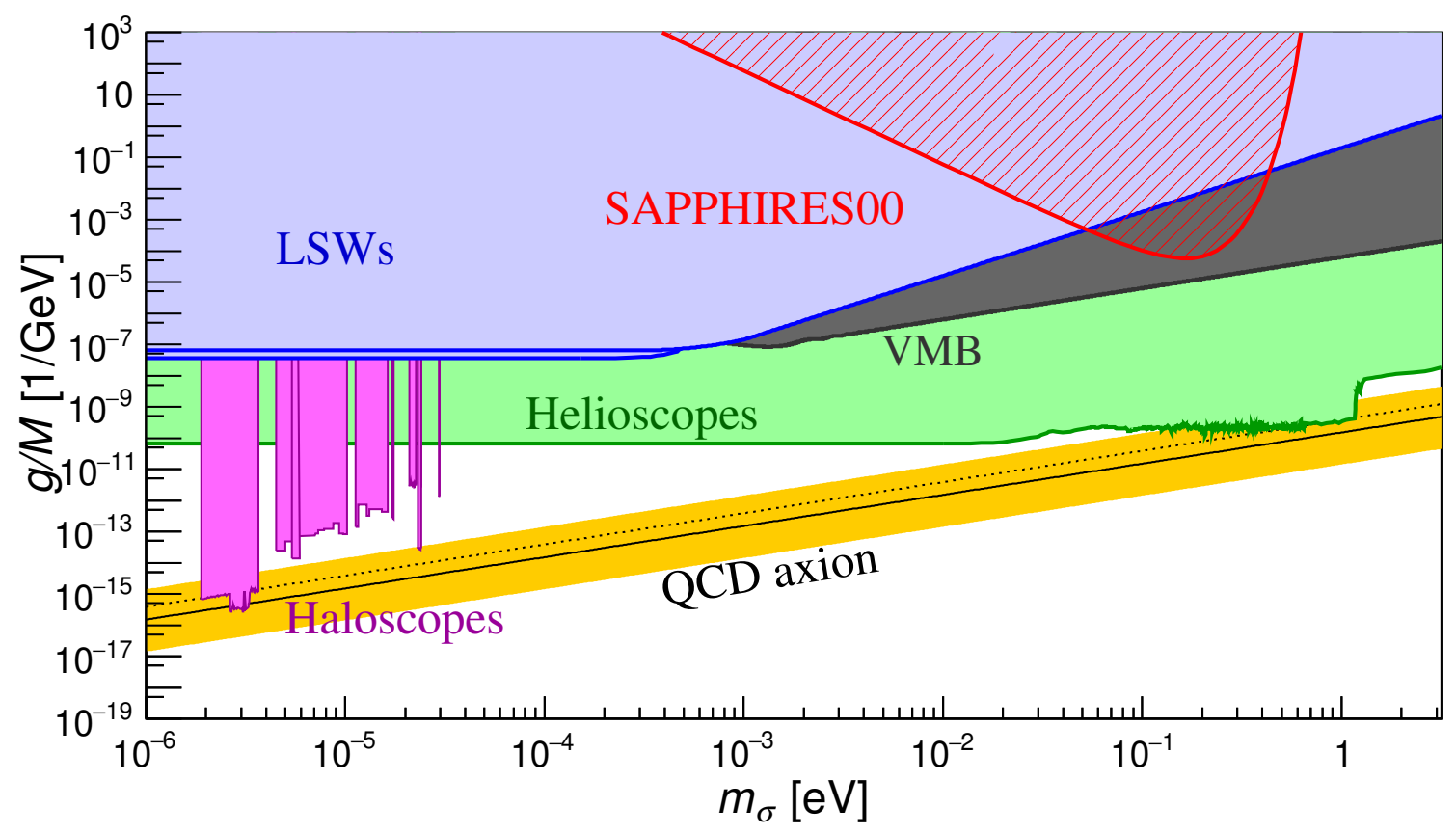

Figure 14. Upper limits on coupling-mass relation for pseudoscalar fields $(\sigma)$ exchanges. The red shaded area labeled "SAPPHIRES00" is the region excluded by this work (stimulated resonant scattering). The yellow band shows the coupling-mass relation based on the QCD axion predicted by the KSVZ model $[19,20]$ with $|E / N-1.95|$ in the range $0.07-7$ with the case of $E / N=0$ (black dotted line). The case of the DFSZ model $[17,18]$ with $E / N=8 / 3$ is also shown by the black solid line. The light-blue area is the region excluded by LSW experiments (OSQAR [34] and ALPS [35]) with respect to pseudoscalar fields. The gray area is the result from the "Vacuum Magnetic Birefringence (VMB)" experiment (PVLAS [36]). The green area shows upper limits from the "Helioscope" experiment (CAST [43-48]). The magenta areas are excluded regions from the "Haloscope" experiments (ADMX [49-52], RBF [53, 54], UF [55], and HAYSTAC [56, 57]).

Technology (MEXT) of Japan. The authors in ELI-NP acknowledge the support by Extreme Light Infrastructure Nuclear Physics Phase II, a project co-financed by the Romanian Government and the European Union through the European Regional Development Fund and the Competitiveness Operational Programme (No. 1/07.07.2016, COP, ID 1334).

\section{A Pulse overlapping factor with different beam diameters}

In order to configure for the actual experimental condition where two beam diameters are different, we replace $\mathcal{D}_{\mathrm{I}}$ in eq. (A.84) of [27] prepared for the application to the common diameter case between creation (subscript $c$ ) and inducing (subscript $i$ ) beams with the following factor $\mathcal{D}_{c+i}$ by taking the diameter difference into account.

$$
\mathcal{D}_{\mathrm{c}+\mathrm{i}}=\frac{1}{2}\left(\frac{2}{\pi}\right)^{\frac{3}{2}} \frac{1}{c^{2}} \frac{\tau_{\mathrm{i}}}{\tau_{\mathrm{c}}} \frac{1}{\sqrt{\tau_{\mathrm{c}}^{2}+2 \tau_{\mathrm{i}}^{2}}} \frac{1}{w_{\mathrm{c} 0}^{2}\left(1-\frac{z_{\mathrm{c} R}^{2}}{z_{\mathrm{i} R}^{2}}\right)}\left[z_{\mathrm{c} R} \tan ^{-1}\left(\frac{z_{\mathrm{i} R}}{z_{\mathrm{c} R}}\right)-R_{\mathrm{ci}} Z_{\mathrm{ci}} \tan ^{-1}\left(\frac{z_{\mathrm{i} R}}{Z_{\mathrm{ci}}}\right)\right]
$$


with

$$
R_{\mathrm{ci}} \equiv \frac{w_{\mathrm{c} 0}^{2} z_{\mathrm{i} R}^{2}+2 w_{\mathrm{i} 0}^{2} z_{\mathrm{cR}}^{2}}{z_{\mathrm{i} R}^{2}\left(w_{\mathrm{c} 0}^{2}+2 w_{\mathrm{i} 0}^{2}\right)}
$$

and

$$
Z_{\mathrm{ci}} \equiv \sqrt{\frac{w_{\mathrm{c} 0}^{2}+2 w_{\mathrm{i} 0}^{2}}{\vartheta_{\mathrm{c} 0}^{2}+2 \vartheta_{\mathrm{i} 0}^{2}}}
$$

using beam diameters $d_{\mathrm{k}}$, beam waists $w_{\mathrm{k} 0}$, and Rayleigh lengths $z_{\mathrm{k} R}$ for $\mathrm{k}=\mathrm{c}$, i defined as

$$
\begin{aligned}
\vartheta_{\mathrm{k} 0} & =\tan ^{-1}\left(\frac{d_{\mathrm{k}}}{2 f}\right), \\
w_{\mathrm{k} 0} & =\frac{\lambda_{\mathrm{k}}}{\pi \vartheta_{\mathrm{k} 0}}, \\
z_{\mathrm{k} R} & =\frac{\pi w_{\mathrm{k} 0}^{2}}{\lambda_{\mathrm{k}}} .
\end{aligned}
$$

We note that $\mathcal{D}_{\mathrm{c}+\mathrm{i}}$ is obtained by integrating the spatiotemporal overlapping factor in eq. (2.3) over the Rayleigh length of the inducing laser which is longer than that of the creation laser in the experimental setup.

Open Access. This article is distributed under the terms of the Creative Commons Attribution License (CC-BY 4.0), which permits any use, distribution and reproduction in any medium, provided the original author(s) and source are credited.

\section{References}

[1] Y. Nambu, Quasiparticles and Gauge Invariance in the Theory of Superconductivity, Phys. Rev. 117 (1960) 648 [INSPIRE].

[2] J. Goldstone, Field Theories with Superconductor Solutions, Nuovo Cim. 19 (1961) 154 [INSPIRE].

[3] R.D. Peccei and H.R. Quinn, CP Conservation in the Presence of Instantons, Phys. Rev. Lett. 38 (1977) 1440 [INSPIRE].

[4] S. Weinberg, A New Light Boson?, Phys. Rev. Lett. 40 (1978) 223 [InSPIRE].

[5] M.A. Shupe et al., Proton Compton Scattering and Neutral Pion Photoproduction at Large Angles, Phys. Rev. Lett. 40 (1978) 271 [INSPIRE].

[6] J. Preskill, M.B. Wise and F. Wilczek, Cosmology of the Invisible Axion, Phys. Lett. B 120 (1983) 127 [INSPIRE].

[7] L.F. Abbott and P. Sikivie, A Cosmological Bound on the Invisible Axion, Phys. Lett. B 120 (1983) 133 [INSPIRE].

[8] M. Dine and W. Fischler, The Not So Harmless Axion, Phys. Lett. B 120 (1983) 137 [INSPIRE].

[9] J.E. Kim, Weak Interaction Singlet and Strong CP Invariance, Phys. Rev. Lett. 43 (1979) 103 [INSPIRE].

[10] M.A. Shifman, A.I. Vainshtein and V.I. Zakharov, Can Confinement Ensure Natural CP Invariance of Strong Interactions?, Nucl. Phys. B 166 (1980) 493 [INSPIRE]. 
[11] Y. Fujii and K. Maeda, The Scalar-Tensor Theory of Gravitation, Cambridge University Press, Cambridge U.K. (2003).

[12] R. Daido, F. Takahashi and W. Yin, The ALP miracle: unified inflaton and dark matter, JCAP 05 (2017) 044 [arXiv: 1702.03284] [INSPIRE].

[13] A. Arvanitaki, S. Dimopoulos, S. Dubovsky, N. Kaloper and J. March-Russell, String Axiverse, Phys. Rev. D 81 (2010) 123530 [arXiv:0905.4720] [INSPIRE].

[14] B.S. Acharya, K. Bobkov and P. Kumar, An M-theory Solution to the Strong CP Problem and Constraints on the Axiverse, JHEP 11 (2010) 105 [arXiv:1004.5138] [INSPIRE].

[15] M. Cicoli, M. Goodsell and A. Ringwald, The type IIB string axiverse and its low-energy phenomenology, JHEP 10 (2012) 146 [arXiv:1206.0819] [INSPIRE].

[16] XENON collaboration, Excess electronic recoil events in XENON1T, Phys. Rev. D 102 (2020) 072004 [arXiv: 2006.09721] [INSPIRE].

[17] M. Dine, W. Fischler and M. Srednicki, A Simple Solution to the Strong CP Problem with a Harmless Axion, Phys. Lett. B 104 (1981) 199 [INSPIRE].

[18] A.R. Zhitnitsky, On Possible Suppression of the Axion Hadron Interactions (in Russian), Sov. J. Nucl. Phys. 31 (1980) 260 [Yad. Fiz. 31 (1980) 497] [INSPIRE].

[19] J.E. Kim, Weak Interaction Singlet and Strong CP Invariance, Phys. Rev. Lett. 43 (1979) 103 [INSPIRE].

[20] M.A. Shifman, A.I. Vainshtein and V.I. Zakharov, Can Confinement Ensure Natural CP Invariance of Strong Interactions?, Nucl. Phys. B 166 (1980) 493 [INSPIRE].

[21] M.M. Miller Bertolami, B.E. Melendez, L.G. Althaus and J. Isern, Revisiting the axion bounds from the Galactic white dwarf luminosity function, JCAP 10 (2014) 069 [arXiv: 1406.7712] [INSPIRE].

[22] A. Ayala, I. Domínguez, M. Giannotti, A. Mirizzi and O. Straniero, Revisiting the bound on axion-photon coupling from Globular Clusters, Phys. Rev. Lett. 113 (2014) 191302 [arXiv: 1406.6053] [INSPIRE].

[23] N. Viaux et al., Neutrino and axion bounds from the globular cluster M5 (NGC 5904), Phys. Rev. Lett. 111 (2013) 231301 [arXiv:1311.1669] [INSPIRE].

[24] M. Giannotti, I.G. Irastorza, J. Redondo, A. Ringwald and K. Saikawa, Stellar Recipes for Axion Hunters, JCAP 10 (2017) 010 [arXiv:1708.02111] [inSPIRE].

[25] L. Di Luzio, M. Giannotti, E. Nardi and L. Visinelli, The landscape of QCD axion models, Phys. Rept. 870 (2020) 1 [arXiv: 2003.01100] [InSPIRE].

[26] Y. Fujii and K. Homma, An approach toward the laboratory search for the scalar field as a candidate of Dark Energy, Prog. Theor. Phys. 126 (2011) 531 [Erratum ibid. 2014 (2014) 089203] [arXiv: 1006.1762] [INSPIRE].

[27] K. Homma and Y. Kirita, Stimulated radar collider for probing gravitationally weak coupling pseudo Nambu-Goldstone bosons, JHEP 09 (2020) 095 [arXiv: 1909. 00983] [INSPIRE].

[28] K. Homma, T. Hasebe and K. Kume, The first search for sub-eV scalar fields via four-wave mixing at a quasi-parallel laser collider, Prog. Theor. Exp. Phys. 2014 (2014) 083C01 [arXiv: 1405.4133] [INSPIRE]. 
[29] T. Hasebe et al., Search for sub-eV scalar and pseudoscalar resonances via four-wave mixing with a laser collider, Prog. Theor. Exp. Phys. 2015 (2015) 073C01 [arXiv:1506.05581] [INSPIRE].

[30] A. Nobuhiro et al., Extended search for sub-eV axion-like resonances via four-wave mixing with a quasi-parallel laser collider in a high-quality vacuum system, Prog. Theor. Exp. Phys. 2020 (2020) 073C01 [arXiv: 2004.10637] [INSPIRE].

[31] S.A.J. Druet and J.-P.E. Taran, Cars spectroscopy, Prog. Quantum Electron. 7 (1981) 1.

[32] K. Homma and Y. Toyota, Exploring pseudo-Nambu-Goldstone bosons by stimulated photon colliders in the mass range $0.1 \mathrm{eV}$ to $10 \mathrm{keV}$, Prog. Theor. Exp. Phys. 2017 (2017) $063 \mathrm{C} 01$ [arXiv: 1701.04282] [INSPIRE].

[33] Particle Data collaboration, Review of Particle Physics (RPP), Phys. Rev. D 86 (2012) 010001 [INSPIRE].

[34] OSQAR collaboration, New exclusion limits on scalar and pseudoscalar axionlike particles from light shining through a wall, Phys. Rev. D 92 (2015) 092002 [arXiv:1506.08082] [INSPIRE].

[35] K. Ehret et al., New ALPS Results on Hidden-Sector Lightweights, Phys. Lett. B 689 (2010) 149 [arXiv: 1004.1313] [INSPIRE].

[36] A. Ejlli et al., The PVLAS experiment: A 25 year effort to measure vacuum magnetic birefringence, Phys. Rept. 871 (2020) 1 [arXiv: 2005.12913] [INSPIRE].

[37] Y. Su et al., New tests of the universality of free fall, Phys. Rev. D 50 (1994) 3614 [Erratum ibid. 51 (1995) 3135] [INSPIRE].

[38] E.G. Adelberger, B.R. Heckel, S.A. Hoedl, C.D. Hoyle, D.J. Kapner and A. Upadhye, Particle Physics Implications of a Recent Test of the Gravitational Inverse Sqaure Law, Phys. Rev. Lett. 98 (2007) 131104 [hep-ph/0611223] [INSPIRE].

[39] D.J. Kapner et al., Tests of the gravitational inverse-square law below the dark-energy length scale, Phys. Rev. Lett. 98 (2007) 021101 [hep-ph/0611184] [INSPIRE].

[40] J. Chiaverini, S.J. Smullin, A.A. Geraci, D.M. Weld and A. Kapitulnik, New experimental constraints on nonNewtonian forces below 100 microns, Phys. Rev. Lett. 90 (2003) 151101 [hep-ph/0209325] [INSPIRE].

[41] S.J. Smullin, A.A. Geraci, D.M. Weld, J. Chiaverini, S.P. Holmes and A. Kapitulnik, New constraints on Yukawa-type deviations from Newtonian gravity at 20 microns, Phys. Rev. D 72 (2005) 122001 [Erratum ibid. 72 (2005) 129901] [hep-ph/0508204] [INSPIRE].

[42] S.K. Lamoreaux, Demonstration of the Casimir force in the 0.6 to 6 micrometers range, Phys. Rev. Lett. 78 (1997) 5 [Erratum ibid. 81 (1998) 5475] [INSPIRE].

[43] CAST collaboration, First results from the CERN Axion Solar Telescope (CAST), Phys. Rev. Lett. 94 (2005) 121301 [hep-ex/0411033] [InSPIRE].

[44] CAST collaboration, An Improved limit on the axion-photon coupling from the CAST experiment, JCAP 04 (2007) 010 [hep-ex/0702006] [INSPIRE].

[45] CAST collaboration, Probing eV-scale axions with CAST, JCAP 02 (2009) 008 [arXiv: 0810.4482] [INSPIRE].

[46] CAST collaboration, CAST search for sub-eV mass solar axions with ${ }^{3}$ He buffer gas, Phys. Rev. Lett. 107 (2011) 261302 [arXiv:1106.3919] [INSPIRE]. 
[47] CAST collaboration, Search for Solar Axions by the CERN Axion Solar Telescope with ${ }^{3} H e$ Buffer Gas: Closing the Hot Dark Matter Gap, Phys. Rev. Lett. 112 (2014) 091302 [arXiv: 1307.1985] [INSPIRE].

[48] CAST collaboration, New CAST Limit on the Axion-Photon Interaction, Nature Phys. 13 (2017) 584 [arXiv : 1705. 02290] [INSPIRE].

[49] ADMX collaboration, An Improved RF cavity search for halo axions, Phys. Rev. D 69 (2004) 011101 [astro-ph/0310042] [INSPIRE].

[50] ADMX collaboration, A SQUID-based microwave cavity search for dark-matter axions, Phys. Rev. Lett. 104 (2010) 041301 [arXiv:0910.5914] [INSPIRE].

[51] ADMX collaboration, A Search for Invisible Axion Dark Matter with the Axion Dark Matter Experiment, Phys. Rev. Lett. 120 (2018) 151301 [arXiv:1804.05750] [INSPIRE].

[52] ADMX collaboration, Piezoelectrically Tuned Multimode Cavity Search for Axion Dark Matter, Phys. Rev. Lett. 121 (2018) 261302 [arXiv:1901.00920] [InSPIRE].

[53] S. De Panfilis et al., Limits on the Abundance and Coupling of Cosmic Axions at $4.5<m_{a}<5.0 \mu \mathrm{eV}$, Phys. Rev. Lett. 59 (1987) 839 [INSPIRE].

[54] W.U. Wuensch et al., Results of a Laboratory Search for Cosmic Axions and Other Weakly Coupled Light Particles, Phys. Rev. D 40 (1989) 3153 [InSPIRE].

[55] C. Hagmann, P. Sikivie, N.S. Sullivan and D.B. Tanner, Results from a search for cosmic axions, Phys. Rev. D 42 (1990) 1297 [inSPIRE].

[56] B.M. Brubaker et al., First results from a microwave cavity axion search at $24 \mu \mathrm{eV}$, Phys. Rev. Lett. 118 (2017) 061302 [arXiv:1610.02580] [INSPIRE].

[57] HAYSTAC collaboration, Results from phase 1 of the HAYSTAC microwave cavity axion experiment, Phys. Rev. D 97 (2018) 092001 [arXiv: 1803.03690] [InSPIRE]. 